\title{
Homogeneous dynamics in a vibrated granular monolayer
}

\section{P Maynar, M I García de Soria and J Javier Brey}

Física Teórica, Universidad de Sevilla, Apartado de Correos 1065, E-41080, Sevilla, Spain

E-mail: maynar@us.es

Received 6 May 2019

Accepted for publication 19 June 2019

Published 16 September 2019

Online at stacks.iop.org/JSTAT/2019/093205

https://doi.org/10.1088/1742-5468/ab3410

\begin{abstract}
A simple model of a vibrated granular monolayer is studied. It consists of inelastic hard spheres confined between two parallel hard plates separated at a distance smaller than twice the diameter of the particles. Both walls are elastic and one of them is vibrating in a sawtooth way. For low densities, a kinetic equation is proposed, from which closed evolution equations for the horizontal and vertical temperatures are derived, assuming spatial homogeneity and that the system is very thin. An excellent agreement between the theoretical predictions and molecular dynamics simulation results is obtained, both for the stationary values and for the dynamics of the temperatures.
\end{abstract}

Keywords: Boltzmann equation, kinetic theory of gases and liquids 


\section{Contents}

1. Introduction 2

2. The model 5

3. Evolution equations for the horizontal and vertical temperatures 9

4. Simulation results $\quad 13$

5. Discussion and conclusions $\quad 19$

Acknowledgments ............................................................. 21

Appendix. Evaluation of some collisional integrals ...................... 21

References $\quad 24$

\section{Introduction}

A granular system is an ensemble of macroscopic particles, grains whose interactions are dissipative. This means that, when two particles interact, part of the kinetic energy of the center of mass of the two particles is transferred to another internal degree of freedom. Granular matter is ubiquitous in nature: from sand dunes to interstellar dust or planetary rings, and they are also relevant because of its technological applications [1]. From a theoretical point of view, granular systems are especially interesting because, due to its dissipative character, they are intrinsically out of equilibrium. A granular system can be fluidized by injecting energy using some kind of forcing, such as vibrating walls or applying a shear. In this so-called fast flow regime, the dynamics is similar to that of a normal fluid as it is, basically, a sequence of binary collisions followed by free streaming of the grains (assuming that the medium in which they are immersed does not affect appreciably its movement). Due to this reminiscence to normal fluids, kinetic equations have been used to study these situations and they have been proved to describe correctly the dynamics of the system [2, 3]. In particular, hydrodynamic equations have been derived in the free cooling case from the Boltzmann or Enskog equations [4, 5], finding explicit expressions for the transport coefficients. Moreover, hydrodynamic equations describe a variety of symmetry-breaking instabilities such as phase-separation instability [6,7], oscillatory instability [8], or thermal granular convection [9] to mention but a few.

A prototypical example of a granular system in the fluidized regime is an ensemble of grains inside a box in which one of the walls, typically the one at the bottom, vibrates injecting energy into the system. In many cases, a stationary state is reached in the long time limit in which the energy lost in collisions is compensated by the energy injected by the wall. In the last two decades, the case of a monolayer of identical spherical grains on a horizontal plate that is vertically vibrated has been widely studied (see, for example, the reviews $[10,11])$. The advantage of this kind of experimental setup with respect to the 'multilayer' case is that, when the height of the system is smaller 
than twice the diameter of the particles, the particles do not jump over each other and it is experimentally possible to follow the motion of all the grains. In addition, states in which the system can be considered to be spatially homogeneous also exist, while for wider systems there are always gradients in the vertical direction. There are many variations of this kind of experiment. Originally, the system is open from above, gravity being the cause of the confinement $[12,13]$. The system can also be confined by a top lid, the distance between plates being much smaller than the horizontal dimensions in such a way that it can be considered quasi-two-dimensional (Q2D) [14-20]. The bottom plate is usually smooth, although rough plates have also been used [14]. Interestingly, they all share a common phenomenology: for a wide range of the parameters, a spatially homogeneous stationary state is reached but, for high enough densities, the system develops a cluster of particles and a final state is reached in which a dense phase coexists with a more dilute and hotter fluid. The instability depends also on the parameters describing the vibrating wall. In addition, depending on the averaged density, the coexistence can be between a solid-like and a liquid-like phase [12-15, 18-20], or between a liquid-like and a gas-like phase [16, 17].

Some simple 2D effective models have been used to try to understand the above phenomenology. The grains are modeled by inelastic hard disks and the wall by some kind of homogeneous energy driving mechanism. In the so-called stochastic thermostat model, the particles are under the action of a stochastic force with vanishing mean value and delta-correlated in time variance [21]. The particles suffer stochastic kicks that can inject energy into the system. In the so-called $\Delta$ model [22], the particles move freely between collisions, but the inelastic collision rule is modified by adding an extra velocity, $\Delta$, to the relative motion pointing outwards in the direction of the collision. Hence, the total kinetic energy of a pair of colliding particles can increase or decrease after a collision. Although the $\Delta$ model seems to better describe the dynamics of the monolayer in homogeneous situations [23], both models fail to explain the phenomenology of the experiments. A homogeneous stationary state is always reached in the long time limit in both cases, i.e. there is no presence of any instability [24-26]. Let us note that more complex 2D models have been studied in which the homogenous stationary state may be unstable. For example, if the stochastic force is multiplicative, in such a way that faster particles receive larger kicks, clusters of particles can arise [27]. Another 2D model that presents phase separation consists of particles with an additional variable that accounts for the kinetic energy stored in the vertical motion [28]. The parameter grows monotonically (following some phenomenological law) until a collision takes place and it is reset to zero. The collision rule depends also on the parameter and, as in the $\Delta$ model, the total kinetic energy of the pair of particles can be increased or decreased in a collision. Although interesting from a theoretical point of view, both models have the disadvantage of depending on some unknown parameters that must be fitted.

When the plates are smooth, it is clear that energy is injected in the vertical direction only, and that it is transferred to the horizontal degrees of freedom via collisions between particles. In order to describe and understand from a microscopic point of view this transference of energy, it is necessary to consider a three-dimensional model. Very recently, the dynamics of an ensemble of elastic hard spheres confined between two parallel hard walls at rest separated a distance smaller than twice the diameter 
of the particles has been studied [29, 30]. For low densities, a closed equation for the one-particle distribution function that takes into account the effects of the confinement was formulated. The proposed Boltzmann-like equation admits an H-theorem [29] and the equilibrium distribution function derived from it agrees with the one obtained by equilibrium statistical mechanics methods [31]. Equations for the horizontal and vertical temperatures were derived finding the specific form of the energy transfer terms and, also, an excellent agreement with molecular dynamics (MD) simulations [30]. The success of kinetic theory to describe confined elastic systems stimulated the study of the model in the inelastic case [32], but with the bottom wall vibrating in a sawtooth way, which always injects energy in the vertical direction. More precisely, in [32] this inelastic model is introduced and phenomenological equations for the vertical and horizontal temperatures are proposed valid for spatially homogeneous states. The equations are supposed to be valid only in the elastic limit because, for the energy transfer terms, the elastic value deduced in [30] was taken. Remarkably, the pressure in the horizontal plane in the stationary state derived from the theory decays monotonically with the density, implying the instability of the homogeneous stationary state if the size of the system exceeds a critical size [32]. In fact, MD simulation results show that, when the homogeneous stationary state is unstable, a dense aggregate surrounded by a dilute hotter gas is formed. The situation is then similar to the results of the experiments reported in $[16,17]$. For spatially homogeneous situations, the predictions of the equations for the horizontal and vertical temperatures agree very well with MD simulation results for mild inelasticities, both for the stationary values and for the dynamics. Out of this range, i.e. for stronger inelasticities, some discrepancies arise.

The objective of this work is to study from a microscopic point of view the inelastic model introduced in [32] in the low density regime. We will follow the same lines stated in the elastic case. Concretely, the first step is to extend the Boltzmann-like equation proposed in $[29,30]$ to inelastic collisions, incorporating also the presence of the vibrating sawtooth wall. The second objective is to derive, from the kinetic equation, the equations for the horizontal and vertical temperatures, assuming spatial homogeneity, but without any restriction about the degree of the inelasticity. The idea is not only to extend the equations for the temperatures proposed in [32] to any inelasticity, but also to have a complete microscopic understanding of them. This study is also motivated by the fact that the characterization of these homogeneous states are essential for the derivation of hydrodynamic equations for spatially inhomogeneous situations. The case in which the two walls are elastic and the system cools down freely will be studied elsewhere [33].

The paper is organized as follows. In the next section, the model is introduced and the kinetic equation is proposed. It is the abovementioned extension of the kinetic equation introduced for elastic systems in [29] to inelastic systems, incorporating also the vibrating wall. In section 3 the equations for the temperatures are obtained from the kinetic equation assuming that the system is spatially homogeneous and that the one-particle distribution function is a Gaussian with two temperatures (the horizontal and vertical temperatures). MD simulations results are presented and compared with the theoretical predictions in section 4. Section 5 contains a summary of the results whose relevance is discussed. Finally, the appendix reports some details of the calculations carried out along the paper. 


\section{The model}

Let us consider an ensemble of $N$ inelastic hard spheres of mass $m$ and diameter $\sigma$ confined between two parallel rectangular-shaped plates of area $A$ separated a distance $H$. It is assumed that $H<2 \sigma$, so that particles can not jump over other particles and the system can be considered Q2D. In the coordinate system we will use, the plates are perpendicular to the $z$ axes and located at $z=0$ and $z=H$, respectively. Particles move freely (gravity is not considered) until there is a particle-particle or particle-wall collision. When there is a binary encounter between two particles with velocities $\mathbf{v}_{1}$ and $\mathbf{v}_{2}$, the postcollisional velocities, $\mathbf{v}_{1}^{\prime}$ and $\mathbf{v}_{2}^{\prime}$, are

$$
\begin{aligned}
& \mathbf{v}_{1}^{\prime} \equiv b_{\hat{\boldsymbol{\sigma}}} \mathbf{v}_{1}=\mathbf{v}_{1}-\frac{1+\alpha}{2}\left(\hat{\boldsymbol{\sigma}} \cdot \mathbf{v}_{12}\right) \hat{\boldsymbol{\sigma}} \\
& \mathbf{v}_{2}^{\prime} \equiv b_{\hat{\boldsymbol{\sigma}} \mathbf{v}_{1}}=\mathbf{v}_{2}+\frac{1+\alpha}{2}\left(\hat{\boldsymbol{\sigma}} \cdot \mathbf{v}_{12}\right) \hat{\boldsymbol{\sigma}}
\end{aligned}
$$

Here we have introduced the operator $b_{\hat{\boldsymbol{\sigma}}}$ that replaces all velocities $\mathbf{v}_{1}$ and $\mathbf{v}_{2}$ appearing to its right by the postcollisional velocities, $\mathbf{v}_{12} \equiv \mathbf{v}_{1}-\mathbf{v}_{2}$ is the relative velocity before the collision and $\hat{\boldsymbol{\sigma}}$ is a unitary vector directed along the line joining the centers of the two particles at contact away from particle 2 . The coefficient $\alpha$ is the coefficient of normal restitution and will be considered to be constant (independent of the relative velocity). It goes in the range $0 \leqslant \alpha \leqslant 1$, being $\alpha=1$ the elastic case. We will always consider inelastic systems, i.e. $\alpha<1$, and periodic boundary conditions in the horizontal directions. The top wall is elastic and at rest, so that when a particle collides with it, it simply reflects its velocity. If a particle with velocity $\mathbf{v}$ collides with the top wall, the postcollisional velocity is

$$
b_{e} \mathbf{v} \equiv v_{x} \mathbf{e}_{x}+v_{y} \mathbf{e}_{y}-v_{z} \mathbf{e}_{z},
$$

where we have introduced the operator $b_{e}$ that transforms the velocity of the particle into its postcollisional velocity. We have also introduced the unitary vectors in the direction of the axes $\left\{\mathbf{e}_{x}, \mathbf{e}_{y}, \mathbf{e}_{z}\right\}$. The bottom wall is modeled by a sawtooth wall of velocity $v_{p}$. Within this model, when there is a collision of a particle with the wall (that is always at $z=0$ ), the particle always sees the wall moving upwards with velocity $v_{p}$. Then, if a particle with velocity $\mathbf{v}$ collides with the bottom wall, the postcollisional velocity is

$$
b_{s} \mathbf{v} \equiv v_{x} \mathbf{e}_{x}+v_{y} \mathbf{e}_{y}+\left(2 v_{p}-v_{z}\right) \mathbf{e}_{z},
$$

where we have introduced the corresponding operator, $b_{s}$. Note that this kind of collision always injects energy into the system and, as in the case of collisions with the top wall, they conserve momentum in the direction parallel to the plates. Since momentum is conserved in the collisions between particles, total horizontal momentum is a constant of the motion. Let us also remark that, in the model, the parameter $v_{p}$ can always be scaled. In effect, let us consider two 'trajectories' of the system, one generated by the initial conditions for the velocities, $\left\{\mathbf{v}_{i}^{(1)}(0)\right\}_{i=1}^{N}$ and a given velocity of the wall, $v_{p}$, and the other generated by $\left\{\mathbf{v}_{i}^{(2)}(0)=K \mathbf{v}_{i}^{(1)}(0)\right\}_{i=1}^{N}$ and $K v_{p}$ with $K$ a given constant. As the collision rules are linear in the velocities, the sequence of collisions is the same 


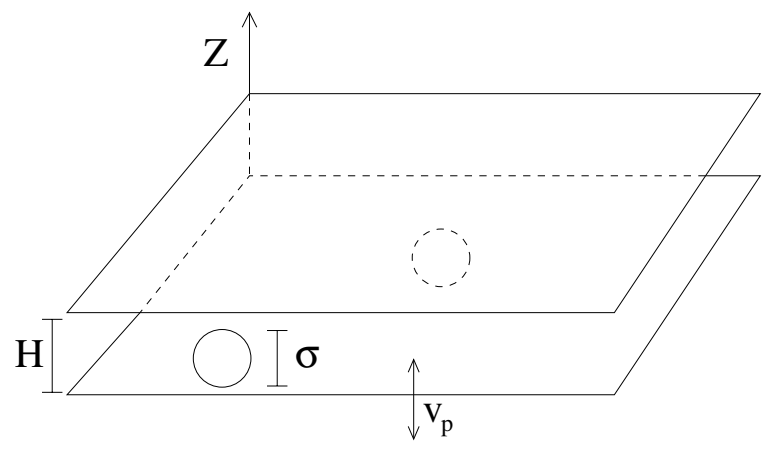

Figure 1. Schematic representation of the model.

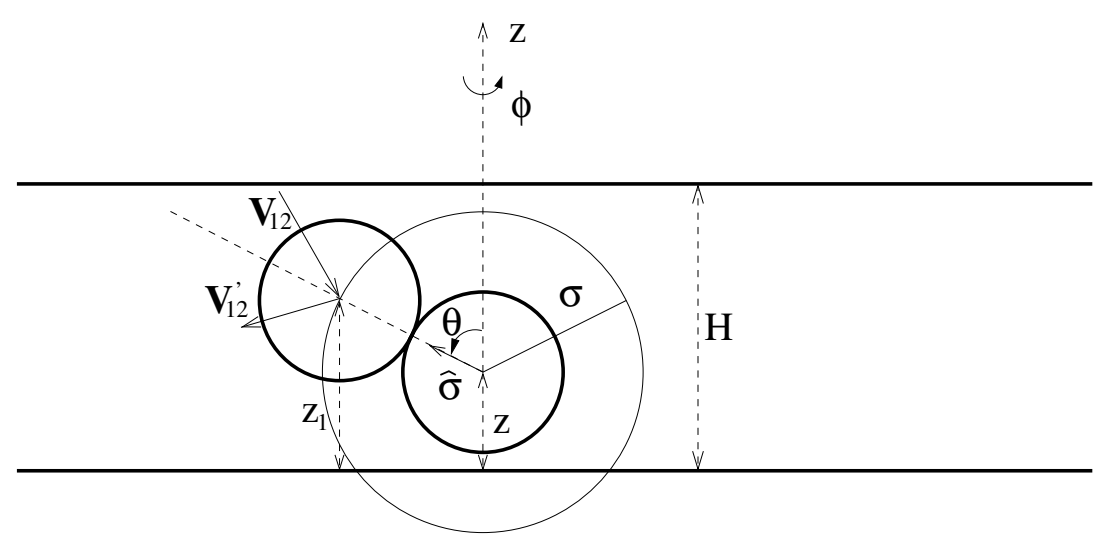

Figure 2. Collision between two inelastic hard spheres in a Q2D system.

in both situations and $\left\{\mathbf{v}_{i}^{(2)}(t)=K \mathbf{v}_{i}^{(1)}(t)\right\}_{i=1}^{N}, \forall t$. Hence, the parameter $v_{p}$ will just fix the energy scale. A scheme of the model is shown in figure 1.

The introduced model is a minimal model to study the experimental situations described in the previous section. Only the essential ingredients are retained: confinement, inelasticity of collisions and energy injection through a vibrating wall. Other aspects such as gravity, friction with the walls, inelasticity of the walls, or friction between particles to mention but a few, are not considered. In any case, the model conditions are expected to hold under some well-defined physical situations, i.e. kinetic energy of the particles much bigger than the maximum potential energy associated to gravity, $m g(H-\sigma)$ with $g$ being the gravity acceleration, and smooth enough particles and walls. Perhaps, the most crude aspect of the model is that only one of the walls is vibrated (the sawtooth wall), while in the experiments the whole box is vibrated sinusoidally.

In the following, a kinetic theory description will be assumed, i.e. a closed description in terms of the one-particle distribution function, $f(\mathbf{r}, \mathbf{v}, t)$, defined as usual as the averaged density of particles with positions around $\mathbf{r}$ and velocities around $\mathbf{v}$ at time $t$. As said, a Boltzmann-like equation describing the dynamics of $f$ of a system of elastic hard spheres confined between two parallel elastic plates has been proposed in [29, 30]. The generalization of the equation to the present model is obtained by modifying the collision rule of both the particle-particle and particle-wall collisions. The derivation of the equation follows standard arguments [34-36]; the time evolution of the one-particle 
distribution function can be decomposed in a free-streaming part, a collisional contribution that takes into account collisions between particles, and a wall contribution that takes into account the collisions between the particles and the walls. In the low density limit, the collisional term can be written in terms of $f$ by assuming molecular chaos, i.e. there are not velocity correlations between the particles that are going to collide, and the equation reads

$$
\left(\frac{\partial}{\partial t}+\mathbf{v}_{2} \cdot \frac{\partial}{\partial \mathbf{r}}\right) f\left(\mathbf{r}, \mathbf{v}_{2}, t\right)=J_{z}[f \mid f]+L_{W} f\left(\mathbf{r}, \mathbf{v}_{2}, t\right) .
$$

Here $J_{z}$ is the collisional contribution

$J_{z}[f \mid f]=\sigma^{2} \int \mathrm{d} \mathbf{v}_{1} \int_{\Omega(z)} \mathrm{d} \hat{\boldsymbol{\sigma}}\left|\mathbf{v}_{12} \cdot \hat{\boldsymbol{\sigma}}\right|\left[\Theta\left(\mathbf{v}_{12} \cdot \hat{\boldsymbol{\sigma}}\right) \alpha^{-2} b_{\hat{\boldsymbol{\sigma}}}^{-1}-\Theta\left(-\mathbf{v}_{12} \cdot \hat{\boldsymbol{\sigma}}\right)\right] f\left(\mathbf{r}+\sigma_{z} \mathbf{e}_{z}, \mathbf{v}_{1}, t\right) f\left(\mathbf{r}, \mathbf{v}_{2}, t\right)$,

where we have introduced the Heaviside step function, $\Theta$, the operator $b_{\hat{\boldsymbol{\sigma}}}^{-1}$ that replaces all velocities appearing to its right by the precollisional velocities, $\mathbf{v}_{1}^{*}$ and $\mathbf{v}_{2}^{*}$,

$$
\begin{aligned}
& \mathbf{v}_{1}^{*} \equiv b_{\hat{\boldsymbol{\sigma}}}^{-1} \mathbf{v}_{1}=\mathbf{v}_{1}-\frac{1+\alpha}{2 \alpha}\left(\hat{\boldsymbol{\sigma}} \cdot \mathbf{v}_{12}\right) \hat{\boldsymbol{\sigma}} \\
& \mathbf{v}_{2}^{*} \equiv b_{\hat{\boldsymbol{\sigma}}}^{-1} \mathbf{v}_{2}=\mathbf{v}_{2}+\frac{1+\alpha}{2 \alpha}\left(\hat{\boldsymbol{\sigma}} \cdot \mathbf{v}_{12}\right) \hat{\boldsymbol{\sigma}}
\end{aligned}
$$

the $z$ component of the vector $\boldsymbol{\sigma} \equiv \sigma \hat{\boldsymbol{\sigma}}, \sigma_{z}$, and the region of integration of $\hat{\boldsymbol{\sigma}}, \Omega(z)$, which depends on the confinement. In spherical coordinates, $\mathrm{d} \hat{\boldsymbol{\sigma}}=\sigma^{2} \sin \theta \mathrm{d} \theta \mathrm{d} \phi$, where $\theta$ and $\phi$ are the polar and azimuthal angles respectively (see figure 2) and the set $\Omega$ can be parametrized as

$$
\Omega(z)=\left\{(\theta, \phi) \mid \theta \in\left(\frac{\pi}{2}-b_{2}(z), \frac{\pi}{2}+b_{1}(z)\right), \phi \in(0,2 \pi)\right\},
$$

with

$$
\begin{aligned}
& b_{1}(z)=\arcsin \left(\frac{z-\sigma / 2}{\sigma}\right) \\
& b_{2}(z)=\arcsin \left(\frac{H-z-\sigma / 2}{\sigma}\right) .
\end{aligned}
$$

Finally, the wall contribution is [36]

$$
L_{W} f(\mathbf{r}, \mathbf{v}, t)=\left[\delta(z-H+\sigma / 2) L_{e}+\delta(z-\sigma / 2) L_{s}\right] f(\mathbf{r}, \mathbf{v}, t)
$$

with

$$
\begin{aligned}
& L_{s} f(\mathbf{r}, \mathbf{v}, t)=\left[\Theta\left(v_{z}-2 v_{p}\right)\left|2 v_{p}-v_{z}\right| b_{s}-\Theta\left(-v_{z}\right)\left|v_{z}\right|\right] f(\mathbf{r}, \mathbf{v}, t), \\
& L_{e} f(\mathbf{r}, \mathbf{v}, t)=\left[\Theta\left(-v_{z}\right)\left|v_{z}\right| b_{e}-\Theta\left(v_{z}\right) v_{z}\right] f(\mathbf{r}, \mathbf{v}, t) .
\end{aligned}
$$

Let us also mention that equation (5) can be directly derived from the first equation of the BBGKY hierarchy by doing the following approximation for the two-particle distribution function, $f_{2}\left(\mathbf{r}_{1}, \mathbf{v}_{1}, \mathbf{r}_{2}, \mathbf{v}_{2}, t\right)$, 


$$
f_{2}\left(\mathbf{r}+\boldsymbol{\sigma}, \mathbf{v}_{1}, \mathbf{r}, \mathbf{v}_{2}, t\right) \approx f\left(\mathbf{r}+\sigma_{z} \mathbf{e}_{z}, \mathbf{v}_{1}, t\right) f\left(\mathbf{r}, \mathbf{v}_{1}, t\right),
$$

for precollisional velocities, i.e. $\mathbf{v}_{12} \cdot \hat{\boldsymbol{\sigma}}<0$, as was done in [37] for elastic hard spheres.

Let us examine the main differences of equation (5) with the 'traditional' Boltzmann equation (without confinement). In the latter, the integration in $\hat{\boldsymbol{\sigma}}$ is over all the solid angles because, at any position, collisions with any orientation are possible. In contrast, in equation (5) this is not the case because, due to the confinement, given a tagged particle, only collisions with the orientation $\hat{\boldsymbol{\sigma}} \in \Omega(z)$ are possible. Otherwise, particle 1 would not fulfill the constrain to be between the two walls. In addition, in the traditional Boltzmann equation it is assumed that the one-particle distribution function does not vary appreciably over distances of the order of $\sigma$, so that $f(\mathbf{r}+\boldsymbol{\sigma}, \mathbf{v}, t) \approx f(\mathbf{r}, \mathbf{v}, t)$. On the other hand, in equation (5) this approximation cannot be done in the $z$ direction because an inconsistent equation would be obtained. In effect, as $\Omega$ depends on $z$, the collisional term, $J_{z}$, depends also on $z$ and, due to the kinetic equation, $f$ does depend explicitly on it. The same occurs with the wall terms. Let us also remark that the same two differences between the confined and traditional Boltzmann equation are present in the elastic case. In fact, the density profile in equilibrium, $n_{e}(z)$, calculated with the confined kinetic equation agrees very well with MD simulation results [29], consistently with the $z$ dependence of the distribution function.

Sometimes it is convenient to change variables in the collisional term from the azimuthal angle, $\theta$, to the $z$ coordinate of the particle that is going to collide with the tagged particle (see figure 2), $z_{1}$,

$$
z_{1}=z+\sigma \cos \theta
$$

In these variables it is

$$
\begin{aligned}
& J_{z}[f \mid f]=\sigma \int \mathrm{d} \mathbf{v}_{1} \int_{\sigma / 2}^{H-\sigma / 2} \mathrm{~d} z_{1}\left|\mathbf{v}_{12} \cdot \hat{\boldsymbol{\sigma}}\left(z, z_{1}, \phi\right)\right|\left[\Theta\left(\mathbf{v}_{12} \cdot \hat{\boldsymbol{\sigma}}\left(z, z_{1}, \phi\right)\right) \alpha^{-2} b_{\hat{\boldsymbol{\sigma}}}^{-1}-\Theta\left(-\mathbf{v}_{12} \cdot \hat{\boldsymbol{\sigma}}\left(z, z_{1}, \phi\right)\right)\right] \\
& f\left(x, y, z_{1}, \mathbf{v}_{1}, t\right) f\left(\mathbf{r}, \mathbf{v}_{2}, t\right)
\end{aligned}
$$

where

$\hat{\boldsymbol{\sigma}}\left(z, z_{1}, \phi\right)=\sqrt{1-\left(\frac{z_{1}-z}{\sigma}\right)^{2}} \cos \phi \mathbf{e}_{x}+\sqrt{1-\left(\frac{z_{1}-z}{\sigma}\right)^{2}} \sin \phi \mathbf{e}_{y}+\frac{z_{1}-z}{\sigma} \mathbf{e}_{z}$.

The advantage of working with $z_{1}$ is that the limits of integration in the collisional term do not depend on $z$ but, on the other hand, the dependance is translated to $\hat{\boldsymbol{\sigma}}$ through equation (18).

As said previously, equation (5) implies a $z$ dependence in the one particle distribution function. Nevertheless, for very dilute systems, it is a good approximation to neglect this dependence, i.e.

$$
f(\mathbf{r}, \mathbf{v}, t) \approx f\left(\mathbf{r}_{\|}, \mathbf{v}, t\right) \equiv \frac{1}{H-\sigma} \int_{\sigma / 2}^{H-\sigma / 2} \mathrm{~d} z f(\mathbf{r}, \mathbf{v}, t),
$$




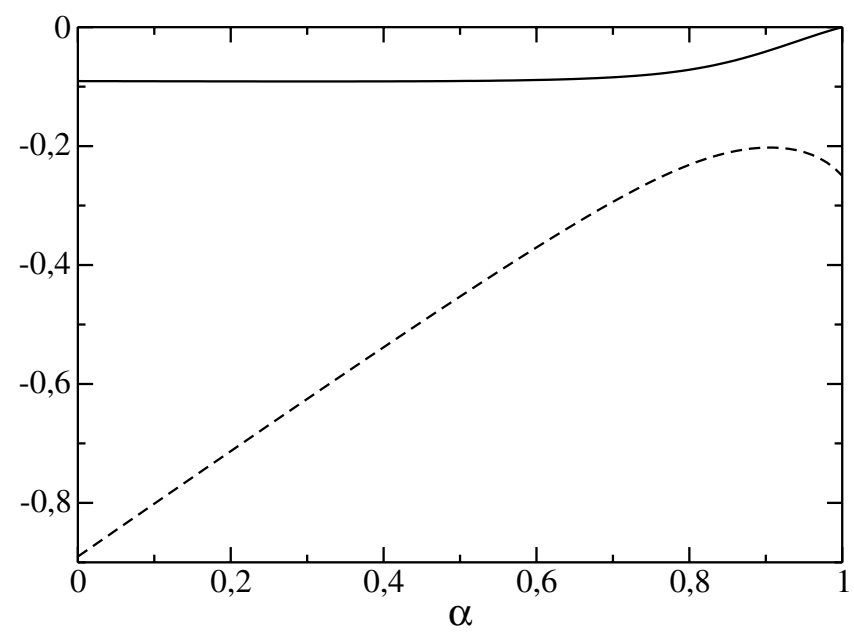

Figure 3. Eigenvalues of the matrix $M, \lambda_{1}$ (solid line) and $\lambda_{2}$ (dashed line), for $\epsilon=0.5$ as a function of the inelasticity.

where we have introduced the parallel component of a vector through $\mathbf{a}_{\|} \equiv a_{x} \mathbf{e}_{x}+a_{y} \mathbf{e}_{y}$. In this situation, by integrating over $z$ in equation (5) and replacing $f(\mathbf{r}, \mathbf{v}, t)$ by $f\left(\mathbf{r}_{\|}, \mathbf{v}, t\right)$ in the collisional operator, $J_{z}$, it is obtained

$$
\left(\frac{\partial}{\partial t}+\mathbf{v}_{2 \|} \cdot \frac{\partial}{\partial \mathbf{r}_{\|}}\right) f\left(\mathbf{r}_{\|}, \mathbf{v}_{2}, t\right)=\frac{1}{H-\sigma} \int_{\sigma / 2}^{H-\sigma / 2} \mathrm{~d} z J_{z}[f \mid f]+\left(L_{e}+L_{s}\right) f\left(\mathbf{r}_{\|}, \mathbf{v}_{2}, t\right),
$$

that is a closed evolution equation for $f\left(\mathbf{r}_{\|}, \mathbf{v}, t\right)$. Of course, equation (20) is fully consistent as no term depends on $z$. The equation is simpler than equation (5) as there have been a reduction in the state variables from $(\mathbf{r}, \mathbf{v})$ to $\left(\mathbf{r}_{\|}, \mathbf{v}\right)$, i.e. the variable $z$ has disappeared although $v_{z}$ remains. In the following, we will assume that the dynamics of the system is given by equation (20).

\section{Evolution equations for the horizontal and vertical temperatures}

Let us consider spatially homogeneous states, i.e. $f\left(\mathbf{r}_{\|}, \mathbf{v}, t\right)=f(\mathbf{v}, t)$. The objective in this section is to derive evolution equations for the horizontal and vertical granular temperatures, $T$ and $T_{z}$, that are defined as

$$
\begin{aligned}
& n T(t)=\frac{m}{2} \int \mathrm{d} \mathbf{v}\left(v_{x}^{2}+v_{y}^{2}\right) f(\mathbf{v}, t), \\
& \frac{n}{2} T_{z}(t)=\frac{m}{2} \int \mathrm{d} \mathbf{v} v_{z}^{2} f(\mathbf{v}, t),
\end{aligned}
$$

where $n$ is the number density, $n \equiv \frac{N}{A(H-\sigma)}$, and we have assumed that there is no macroscopic velocity field, i.e. $\int \operatorname{d} \mathbf{v} \mathbf{v} f(\mathbf{v}, t)=0$. To proceed, we take velocity moments in the kinetic equation. Multiplying equation (20) by $\frac{m}{2}\left(v_{x}^{2}+v_{y}^{2}\right)$ and integrating in the velocity, it is obtained 


$$
n \frac{\mathrm{d} T}{\mathrm{~d} t}=\frac{1}{H-\sigma} \int_{\sigma / 2}^{H-\sigma / 2} \mathrm{~d} z \int \mathrm{d} \mathbf{v} \frac{m}{2}\left(v_{x}^{2}+v_{y}^{2}\right) J_{z}[f \mid f] .
$$

Note that the walls contribution trivially vanishes since there is not energy injection in the horizontal direction. Analogously, multiplying equation (20) by $\frac{m}{2} v_{z}^{2}$ and integrating in the velocity, it is obtained that

$$
\frac{n}{2} \frac{\mathrm{d} T_{z}}{\mathrm{~d} t}=\frac{1}{H-\sigma} \int_{\sigma / 2}^{H-\sigma / 2} \mathrm{~d} z \int \mathrm{d} \mathbf{v} \frac{m}{2} v_{z}^{2} J_{z}[f \mid f]+\frac{m}{2(H-\sigma)} \int \mathrm{d} \mathbf{v} v_{z}^{2} L_{s} f(\mathbf{v}, t) .
$$

In this case, the top wall does not contribute (it is at rest), while the bottom wall contribution is expressed in terms of the $L_{s}$ operator.

To close equations (23) and (24), we have to express the velocity moments of the collisional term in terms of the horizontal and vertical temperatures. In order to do it, we will assume that the distribution function is, for all times, very close to a Maxwellian distribution characterized by the temperatures in the vertical and horizontal directions, i.e.

$$
f(\mathbf{v}, t)=\frac{n}{\pi^{3 / 2} w^{2}(t) w_{z}(t)} \mathrm{e}^{-\frac{v_{x}^{2}}{w^{2}(t)}-\frac{v_{y}^{2}}{w^{2}(t)}-\frac{v_{z}^{2}}{w_{z}^{2}(t)}}
$$

where we have introduced the thermal velocities in the horizontal and vertical direction, $w$ and $w_{z}$, through

$$
\begin{aligned}
& \frac{m}{2} w^{2}(t) \equiv T(t), \\
& \frac{m}{2} w_{z}^{2}(t) \equiv T_{z}(t) .
\end{aligned}
$$

The validity of the approximation will be confirmed by MD simulation results. The calculation is done in the appendix, obtaining

$$
\begin{gathered}
\frac{1}{H-\sigma} \int_{\sigma / 2}^{H-\sigma / 2} \mathrm{~d} z \int \mathrm{d} \mathbf{v} \frac{m}{2}\left(v_{x}^{2}+v_{y}^{2}\right) J_{z}[f \mid f]=\frac{2 \sqrt{2 \pi}(1+\alpha) n^{2} \sigma^{2}}{\epsilon} \int_{0}^{\epsilon} \mathrm{d} y(\epsilon-y)\left(1-y^{2}\right) \\
\left\{\frac{1+\alpha}{2}\left[w^{2}\left(1-y^{2}\right)+w_{z}^{2} y^{2}\right]^{3 / 2}-w^{2}\left[w^{2}\left(1-y^{2}\right)+w_{z}^{2} y^{2}\right]^{1 / 2}\right\}, \\
\frac{1}{H-\sigma} \int_{\sigma / 2}^{H-\sigma / 2} \mathrm{~d} z \int \mathrm{d} \mathbf{v} \frac{m}{2} v_{z}^{2} J_{z}[f \mid f]=\frac{2 \sqrt{2 \pi}(1+\alpha) n^{2} \sigma^{2}}{\epsilon} \int_{0}^{\epsilon} \mathrm{d} y(\epsilon-y) y^{2} \\
\left\{\frac{1+\alpha}{2}\left[w^{2}\left(1-y^{2}\right)+w_{z}^{2} y^{2}\right]^{3 / 2}-w_{z}^{2}\left[w^{2}\left(1-y^{2}\right)+w_{z}^{2} y^{2}\right]^{1 / 2}\right\},
\end{gathered}
$$

where we have introduced the dimensionless parameter $\epsilon \equiv \frac{H-\sigma}{\sigma}$. Although the above integrals can be evaluated exactly, their expressions are very long and we prefer to leave them in the more compact given form. The wall contribution is also evaluated in the appendix, obtaining 


$$
\frac{m}{2(H-\sigma)} \int \mathrm{d} \mathbf{v} v_{z}^{2} L_{s} f(\mathbf{v}, t)=\frac{n v_{p} T_{z}}{\epsilon \sigma}
$$

that coincides with the exact result derived in [38] (it is $v_{p}$ times the pressure of the granular system just above the vibrating wall in the direction perpendicular to it).

In the following, we will perform an expansion of the collisional terms to third order in $\epsilon$. The reason is that, in this case, the obtained expressions are easier to handle and the several terms can be understood intuitively in a simple way. To this order, the equations are

$$
\begin{aligned}
& \frac{\mathrm{d} T}{\mathrm{~d} t}=\sqrt{\pi}(1+\alpha) \epsilon n \sigma^{2} \sqrt{\frac{T}{m}}\left[-(1-\alpha) T+\epsilon^{2}\left(-\frac{5 \alpha-1}{12} T+\frac{3 \alpha+1}{12} T_{z}\right)\right], \\
& \frac{\mathrm{d} T_{z}}{\mathrm{~d} t}=\frac{2}{3} \sqrt{\pi}(1+\alpha) \epsilon^{3} n \sigma^{2} \sqrt{\frac{T}{m}}\left(\frac{1+\alpha}{2} T-T_{z}\right)+\frac{2 v_{p} T_{z}}{\epsilon \sigma} .
\end{aligned}
$$

Let us briefly analyze the structure of the equations. First, let us mention that, as the equations are obtained as an expansion in powers of $\epsilon$, they are only valid for very thin systems. Moreover, the considered third order in $\epsilon$, is the lowest order consistent with the existence of a non-trivial stationary state. In effect, neglecting the $\epsilon^{3}$ terms, the only stationary state is the one with vanishing temperatures. The first order in $\epsilon$ term in equation (31) is, essentially, the cooling term due to the inelasticity of the collisions. Actually, it coincides with the cooling term of a free evolving hard disks system in the Gaussian approximation [39]. The $\epsilon^{3}$ terms in the equations describe energy transfer from the vertical to the horizontal degrees of freedom due to collisions between particles. Finally, the last term in equation (32) is the energy injection term due to collisions of particles with the bottom wall. Hence, the dynamics can be summarized as follows: particle-bottom wall collisions inject energy in the vertical direction and particle-particle collisions transfer energy form the vertical to the horizontal directions and also dissipate it. Let us also mention that, for small inelasticities, equations (31) and (32) reduce to the ones used in [32], and in the elastic case $\left(\alpha=1\right.$ with $\left.v_{p}=0\right)$ to the ones of [30].

Equations (31) and (32) present a simpler form when expressed in the dimensionless time scale, $s(t)$, defined through

$$
s(t)=\sqrt{\frac{\pi}{2}}(1+\alpha) n \sigma^{2} \epsilon \int_{0}^{t} \mathrm{~d} t^{\prime} w\left(t^{\prime}\right)
$$

which is proportional to the number of collisions per particle in the time interval $(0, t)$. In effect, let us introduce the dimensionless temperatures

$$
\begin{aligned}
& \widetilde{T} \equiv \frac{T}{m v_{p}^{2}}, \\
& \widetilde{T}_{z} \equiv \frac{T_{z}}{m v_{p}^{2}} .
\end{aligned}
$$

The evolution equations are 


$$
\begin{aligned}
& \frac{\mathrm{d} \widetilde{T}}{\mathrm{~d} s}=-(1-\alpha) \widetilde{T}+\epsilon^{2}\left(-\frac{5 \alpha-1}{12} \widetilde{T}+\frac{3 \alpha+1}{12} \widetilde{T}_{z}\right) \\
& \frac{\mathrm{d} \widetilde{T}_{z}}{\mathrm{~d} s}=\frac{2}{3} \epsilon^{2}\left(\frac{1+\alpha}{2} \widetilde{T}-\widetilde{T}_{z}\right)+\frac{2}{\sqrt{\pi}(1+\alpha) n \sigma^{3} \epsilon^{2}} \frac{\widetilde{T}_{z}}{\sqrt{\widetilde{T}}}
\end{aligned}
$$

which do not depend on $v_{p}$ as a consequence of the property mentioned in section 2 that $v_{p}$ only sets the energy scale.

From equations (31) and (32) (or equations (36) and (37)) the stationary temperatures, $T_{s}$ and $T_{z, s}$, can be easily calculated. From the horizontal temperature equation, it follows that the ratio of stationary temperatures is

$$
\gamma \equiv \frac{T_{z, s}}{T_{s}}=\frac{12(1-\alpha)+(5 \alpha-1) \epsilon^{2}}{(3 \alpha+1) \epsilon^{2}}
$$

that is density independent. The stationary horizontal temperature is smaller than the stationary vertical temperature for $0 \leqslant \alpha<1$ and $0<\epsilon<1$, with equipartition holding in the elastic limit, i.e. $\lim _{\alpha \rightarrow 1} \gamma=1$. From the vertical temperature equation, the stationary horizontal temperature is obtained as

$$
T_{s}=\left[\frac{3 \gamma}{\sqrt{\pi}(1+\alpha)\left(\gamma-\frac{1+\alpha}{2}\right) \epsilon^{3} \tilde{n} \sigma^{2}}\right]^{2} m v_{p}^{2},
$$

where the effective $2 \mathrm{D}$ density, $\tilde{n} \equiv \frac{N}{A}$, has been introduced. As the dimensionless parameters $\epsilon$ and $\tilde{n} \sigma^{2}$ are supposed to be small, the thermal horizontal and vertical velocities are much bigger than the velocity of the wall, $v_{p}$. This can be intuitively understood since the thinner and the more dilute is the system, the bigger is the ratio between the particle-wall collisions and particle-particle collisions. As the collisions with the sawtooth wall always inject energy, the temperature increases when $\epsilon$ and/or $\tilde{n} \sigma^{2}$ decrease.

Let us consider now situations in which both temperatures are close to their respective stationary values. Then, to linear order, the deviations $\delta T \equiv T-T_{s}$ and $\delta T_{z} \equiv T_{z}-T_{z, s}$ obey the following set of linear differential equations

$$
\frac{\mathrm{d}}{\mathrm{d} s}\left[\begin{array}{l}
\delta T \\
\delta T_{z}
\end{array}\right]=M\left[\begin{array}{l}
\delta T \\
\delta T_{z}
\end{array}\right]
$$

where we have introduced the matrix

$$
M=\left[\begin{array}{cc}
-1+\alpha-\frac{5 \alpha-1}{12} \epsilon^{2} & \frac{3 \alpha+1}{12} \epsilon^{2} \\
\left(\frac{1+\alpha}{2}-\frac{\gamma}{3}\right) \epsilon^{2} & -\frac{1+\alpha}{3 \gamma} \epsilon^{2}
\end{array}\right] .
$$

The solution of the system is

$$
\left[\begin{array}{l}
\delta T(s) \\
\delta T_{z}(s)
\end{array}\right]=\sum_{i=1}^{2} \mathbf{v}_{1} \cdot\left[\begin{array}{l}
\delta T(0) \\
\delta T_{z}(0)
\end{array}\right] \mathbf{u}_{i} \mathrm{e}^{\lambda_{i} s}
$$


where $\left\{\lambda_{1}, \lambda_{2}\right\},\left\{\mathbf{u}_{1}, \mathbf{u}_{2}\right\}$ and $\left\{\mathbf{v}_{1}, \mathbf{v}_{2}\right\}$ are the eigenvalues, right eigenfunctions and left eigenfunctions of $M$ respectively. The eigenvalues are always negative so that the system of differential equations given by equation (40) is linearly stable. In figure 3 the eigenvalues, $\lambda_{1}$ (solid line) and $\lambda_{2}$ (dashed line), are plotted for $\epsilon=0.5$ as a function of the inelasticity. It is found that the two eigenvalues are always separated, one of them, $\lambda_{1}$, being the slowest and vanishing in the elastic limit. The same kind of behavior is obtained for a wide range of the values of the parameters although, for $\epsilon \sim 0.8$ the eigenvalues cross each other at $\alpha \sim 0.4$. In any case, it is not clear that for such a height equations (31) and (32) describe correctly the dynamics of the system. The fact that the eigenvalues are always well separated implies that there is a time scale, that will be called a 'homogeneous hydrodynamic' time scale, in which the dynamics is governed by the slowest mode, $\lambda_{1}$. In this regime the two temperatures are related through

$$
\delta T_{z} \approx q \delta T
$$

with

$$
q \equiv \frac{u_{11}}{u_{12}}=\frac{12\left(\lambda_{1}+1-\alpha\right)+(5 \alpha-1) \epsilon^{2}}{(3 \alpha+1) \epsilon^{2}} .
$$

In the next section, we will see that the homogeneous hydrodynamic regime is not an exclusive characteristic of the linear case, but that it also arises in general.

\section{Simulation results}

To check the validity of the results of the previous section, we have carried out MD simulations of our model using the event-driven algorithm [40]. The instantaneous positions and velocities of all the grains have been measured for different values of the parameters, starting with different initial conditions. All the simulations are run with $N=500$ and $\tilde{n} \sigma^{2}=0.03$, taking the mass of the grains as the unit of mass. In most of the simulations $v_{p}=0.001\left[\frac{T_{0}}{m}\right]^{1 / 2}$ with $T_{0} \equiv T(0)$ the initial horizontal temperature that is taken to be unity. If not, it is explicitly indicated. The initial condition was taken to be an anisotropic Gaussian of the form given by equation (25). We have checked that the system stays for all times spatially homogeneous in the horizontal direction and that, after a transient, a steady state is reached. Let us first focus on the properties of this stationary state. In this case, all the results are generated with one trajectory, averaging over a time period of about 50000 total collisions (particle-particle and particle-wall) per particle once the steady state is reached. In figure 4 (color online), the simulation results for the logarithm of the stationary marginals velocity distribution functions, $f_{s, x}\left(v_{x}\right) \equiv \int \mathrm{d} v_{y} \int \mathrm{d} v_{z} f_{s}(\mathbf{v})$ (black circles) and $f_{s, z}\left(v_{z}\right) \equiv \int \mathrm{d} v_{x} \int \mathrm{d} v_{y} f_{s}(\mathbf{v})$ (red squares), are plotted for $\epsilon=0.5$ and $\alpha=0.8$ as a function of $v_{x}$ and $v_{z}$ respectively. The black dashed line and point red line are the corresponding quadratic interpolations. It is found that they can be very well fitted by Gaussians as was said in the previous section. Similar results are found for other values of the parameters.

In figure 5 the ratio between the stationary temperatures, $\gamma \equiv \frac{T_{z, s}}{T_{s}}$, is plotted for $\epsilon=0.5$ (circles) and $\epsilon=0.2$ (squares), and the coefficient of normal restitution in the 


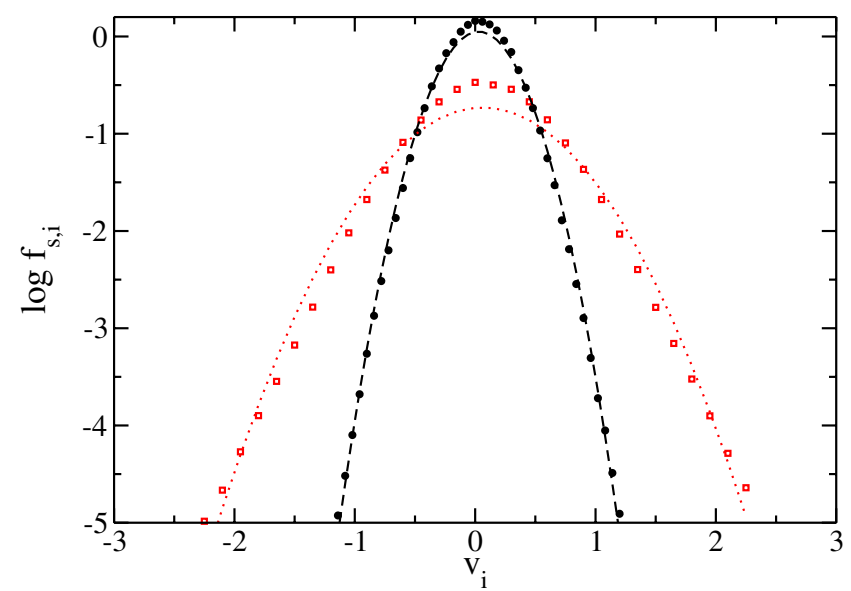

Figure 4. Simulation results for the logarithm of the stationary marginals velocity distribution functions (color online), $f_{s, x}\left(v_{x}\right)$ (black circles) and $f_{s, z}\left(v_{z}\right)$ (red squares) for $\epsilon=0.5$ and $\alpha=0.8$ as a function of $v_{x}$ and $v_{z}$ respectively. The black dashed line and point red line are the corresponding quadratic interpolations.

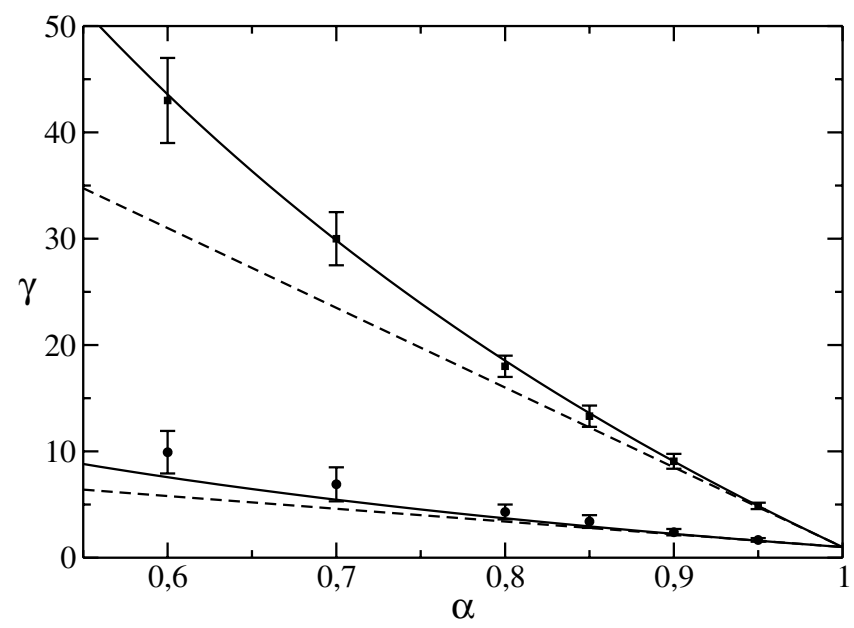

Figure 5. Ratio between the stationary temperatures, $\gamma$, for $\epsilon=0.5$ (circles) and $\epsilon=0.2$ (squares) as a function of the inelasticity. The solid lines are the corresponding theoretical prediction given by equation (38) and the dashed lines are the quasielastic theoretical prediction of [32].

range $0.6 \leqslant \alpha \leqslant 0.95$. The error bars have been calculated from the dispersion of the temperatures measured once the stationary state has been reached. The solid lines are the corresponding theoretical predictions given by equation (38), finding an excellent agreement with the MD simulations results for the whole range of inelasticities. This is remarkable as there is not any fitting parameter. It can be appreciated that the agreement in the $\epsilon=0.2$ case is better than for $\epsilon=0.5$. This was expected since the theory is implemented by a power expansion around $\epsilon=0$. The quasielastic theoretical predictions of [32] for $\epsilon=0.2$ and $\epsilon=0.5$ are also plotted (dashed lines). Although this prediction captures the tendency of the data, it is clearly seen that the new prediction 


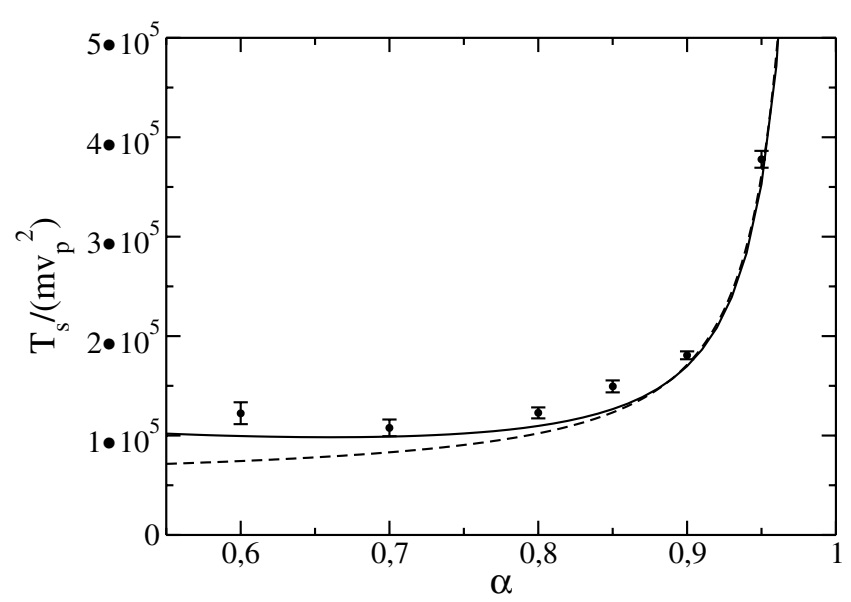

Figure 6. Stationary horizontal temperature scaled with $m v_{p}^{2}$ for $\epsilon=0.5$. The circles are the simulation results and the solid line is the theoretical prediction given by equation (39). The dashed line is the quasielastic theoretical prediction of [32].

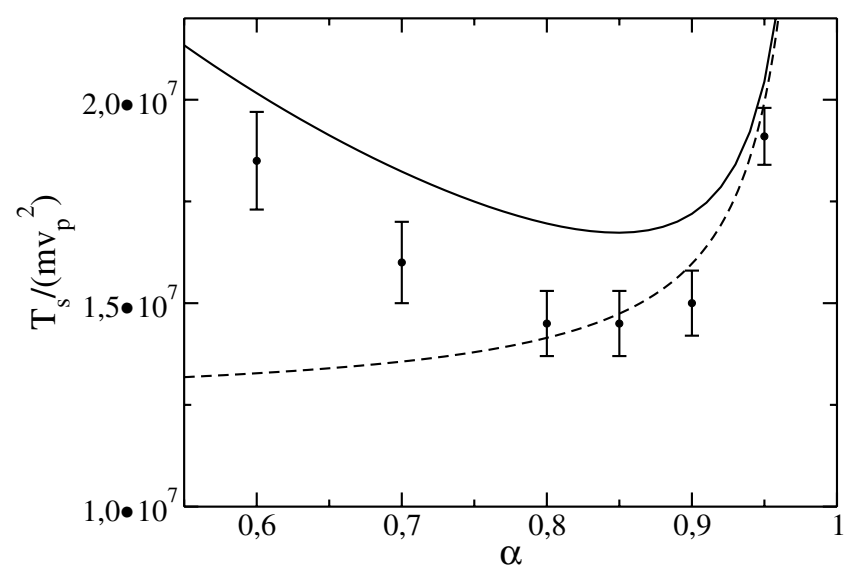

Figure 7. Stationary horizontal temperature scaled with $m v_{p}^{2}$ for $\epsilon=0.2$. The circles are the simulation results and the solid line is the theoretical prediction given by equation (39). The dashed line is the quasielastic theoretical prediction of [32].

given by equation (38) improves considerably the agreement with the simulation results, specially for strong inelasticities.

In figure 6 we have plotted the stationary horizontal temperature scaled with $m v_{p}^{2}$ for $\epsilon=0.5$ as a function of $\alpha$. The circles are the MD simulation results and the solid line is the theoretical prediction given by equation (39). The dashed line is the quasielastic prediction calculated in [32]. The error bars are evaluated as in figure 5. Again, the agreement between the theoretical prediction and the simulation results is very good, and the 'inelastic' prediction improves the agreement with respect to the quasielastic one. Let us remark that the quasielastic prediction of the stationary horizontal temperature decays monotonically with the inelasticity, while equation (39) predicts an enhanced one that actually is observed in the simulations. The same is plotted in figure 7 for $\epsilon=0.2$. Here, it seems that the agreement with the quasielastic prediction is 


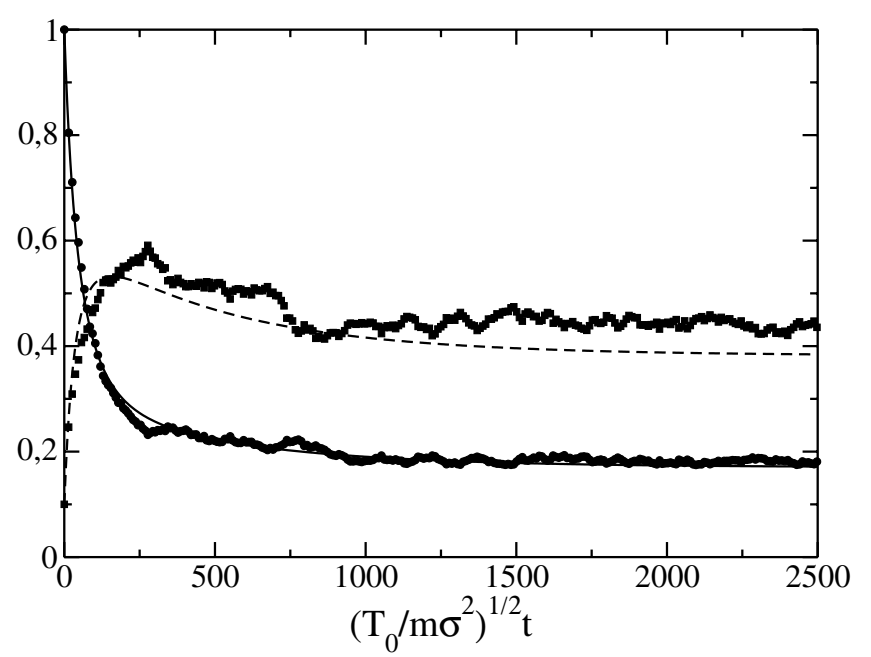

Figure 8. Time evolution of the horizontal (circles) and vertical (squares) temperatures for $\epsilon=0.5$ and $\alpha=0.9$. The solid and dashed lines are the numerical solution of equations (31) and (32) for the horizontal and vertical temperatures respectively.

better than with equation (39) for $0.8<\alpha<0.9$, although it is clear that this is not the case for strong inelasticities. In fact, the minimum of the stationary temperature measured in the simulation is around $\alpha \sim 0.85$, as predicts equation (39). Let us also stress that $\frac{T_{s}}{m v_{p}^{2}} \sim 10^{5}$ for $\epsilon=0.5$ and $\frac{T_{s}}{m v_{p}^{2}} \sim 10^{7}$ for $\epsilon=0.2$, i.e. the thermal horizontal velocity is much larger than the velocity of the wall. This was commented in the previous section and it is a consequence of the fact that there are two dimensionless parameters, $\epsilon$ and $\tilde{n} \sigma^{2}$, which contribute to the increase of $T_{s}$ (see equation (39)).

Let us examine now if equations (31) and (32) correctly describe the time evolution of the system. In figure 8 we have plotted the MD simulation results of the time evolution of the horizontal (circles) and vertical (squares) temperatures. The dimensionless height of the system is $\epsilon=0.5$ and $\alpha=0.9$, and the initial condition was taken to be a Gaussian with $T_{z}(0)=0.1 T_{0}$. The solid and dashed lines are the numerical solution of equations (31) and (32) for the horizontal and vertical temperatures, respectively. The agreement with the simulation results is excellent for the whole time evolution. Note that there is a time window, around $(100,300)$ in the dimensionless time scale $\left[\frac{T_{0}}{m}\right]^{1 / 2} \frac{t}{\sigma}$, in which the degree of freedom with the highest granular temperature (the vertical one) heats up while the degree of freedom with the lowest granular temperature (the horizontal one) cools down. A similar good agreement between the MD simulation results and the theoretical predictions is obtained for other values of the parameters and initial conditions.

For given values of the parameters, we have studied the dynamics of the system starting with different initial conditions. The initial condition was always a Gaussian with two temperatures. It is found that, after a transient, the system reaches a regime in which the horizontal and vertical temperatures are related, independently of the initial condition. This is the generalization to the non-linear case of the homogeneous hydrodynamic regime studied in the linear case in the previous section. In effect, in figure 9 the vertical temperature is plotted as a function of the horizontal temperature for $\alpha=0.9$ 


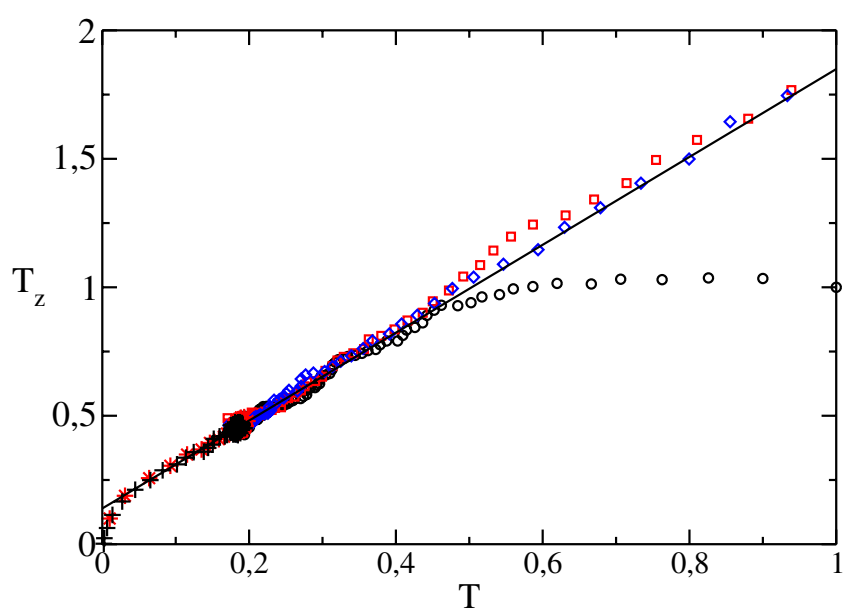

Figure 9. Vertical temperature versus horizontal temperature for $\alpha=0.9$ and $\epsilon=0.5$. The initial conditions are $\left(T(0), T_{z}(0)\right)=\left(T_{0}, T_{0}\right)$ (black circles), $\left(T(0), T_{z}(0)\right)=\left(10 T_{0}, 10 T_{0}\right)$ (redsquares), $\left(T(0), T_{z}(0)\right)=\left(20 T_{0}, 20 T_{0}\right)$ (bluediamonds), $\left(T(0), T_{z}(0)\right)=\left(0.002 T_{0}, 0.002 T_{0}\right)$ (black pluses), and $\left(T(0), T_{z}(0)\right)=\left(0.01 T_{0}, 0.01 T_{0}\right)$ (red stars). $T_{0}$ is taken as unity. The solid line is the theoretical prediction for the hydrodynamic regime close to the stationary state.

and $\epsilon=0.5$ starting with different initial conditions. The value of the velocity of the wall is, in this case, the same for all the initial conditions, $v_{p}=0.001\left(\frac{T_{0}}{m}\right)^{1 / 2}, T_{0}$ being the initial horizontal temperature of one of the simulations that, as before, is taken to be unity. The initial conditions are (color online) $\left(T(0), T_{z}(0)\right)=\left(T_{0}, T_{0}\right)$ (black circles), $\left(T(0), T_{z}(0)\right)=\left(10 T_{0}, 10 T_{0}\right)$ (red squares), $\left(T(0), T_{z}(0)\right)=\left(20 T_{0}, 20 T_{0}\right)$ (blue diamonds), $\left(T(0), T_{z}(0)\right)=\left(0.002 T_{0}, 0.002 T_{0}\right)$ (black pluses), and $\left(T(0), T_{z}(0)\right)=\left(0.01 T_{0}, 0.01 T_{0}\right)$ (red stars). It is seen that, after a transient, the curves collapse to a single curve through which the stationary state is reached $\left(T_{s} \approx 0.18 T_{0}\right.$ and $\left.T_{z, s} \approx 0.45 T_{0}\right)$. In the figure, the universal curve in the linear regime is also plotted, $T_{z}^{(H)}(T) \approx T_{z, s}+q\left(T-T_{s}\right)$, with the values of the stationary temperatures taken from the simulations and the value of $q$ taken from the theoretical prediction given by equation (44). As it can be seen, this curve is a good approximation for the region in which the temperatures collapse (the universal homogeneous hydrodynamic regime) even for temperatures that are far apart the stationary state, and for which the linear equations, equations (40), are not supposed to be valid. Why can $T_{z}^{(H)}(T)$ be approximated by its linear expansion around the stationary state out of the linear regime? This can be understood, at least for $T>T_{s}$. In effect, for high temperatures (compared to $T_{s}$ ), the system does not 'feel' the vibrating wall and may evolve cooling freely. In this case, the dynamics of the temperatures is linear in the $s$ variable

$$
\frac{\mathrm{d}}{\mathrm{d} s}\left[\begin{array}{c}
T \\
T_{z}
\end{array}\right]=M_{f}\left[\begin{array}{c}
T \\
T_{z}
\end{array}\right],
$$

where we have introduced the matrix

$$
M_{f}=\left[\begin{array}{cc}
-1+\alpha-\frac{5 \alpha-1}{12} \epsilon^{2} & \frac{3 \alpha+1}{12} \epsilon^{2} \\
\frac{1+\alpha}{3} \epsilon^{2} & -\frac{2}{3} \epsilon^{2}
\end{array}\right] .
$$




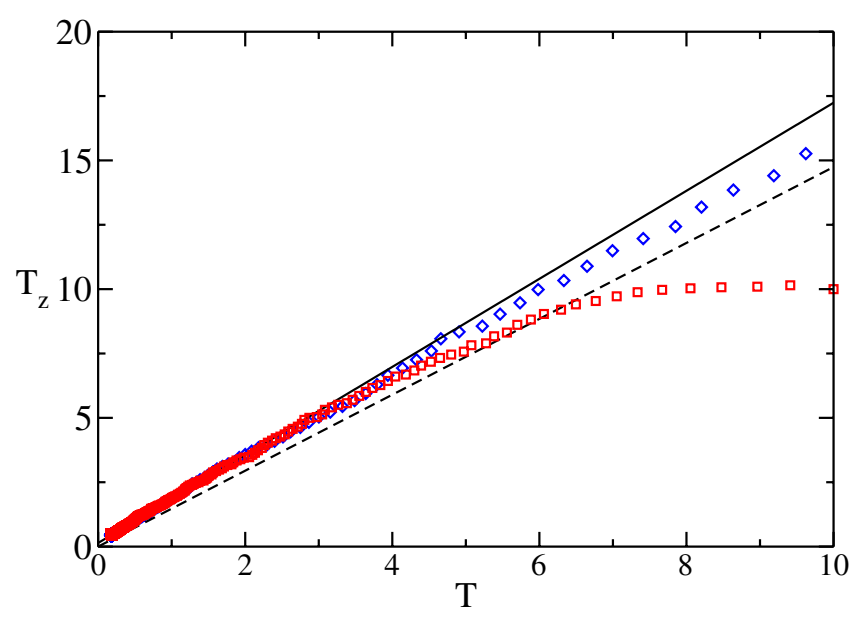

Figure 10. Vertical temperature versus horizontal temperature for $\alpha=0.9$ and $\epsilon=0.5$. The initial conditions are $\left(T(0), T_{z}(0)\right)=\left(10 T_{0}, 10 T_{0}\right)$ (red squares) and $\left(T(0), T_{z}(0)\right)=\left(20 T_{0}, 20 T_{0}\right)$ (blue diamonds). The solid and dashed lines are the theoretical predictions close to the stationary state and for the free cooling case respectively, both in the hydrodynamic regime.

The structure of the matrix $M_{f}$ is similar to $M$, in the sense that the two eigenvalues are the negative one of them dominating the dynamics in the long time limit, so that $T_{z} \approx q_{f} T_{z}$ in this regime [33]. The coefficient $q_{f}$ can be calculated in a similar fashion that $q$ is obtaining, in addition, that they are very close. Hence, coming back to the vibrating case again, the universal curve goes from the free cooling behavior for high temperatures to the linear regime close to the stationary state. As the two functions are similar and the transition from one case to the other is expected to be smooth, the function $T_{z}^{(H)}(T) \approx T_{z, s}+q\left(T-T_{s}\right)$ is expected to be a good approximation for $T_{z}^{(H)}(T)$ in a much wider regime. In figure 10 the results for the initial conditions $\left(T(0), T_{z}(0)\right)=\left(10 T_{0}, 10 T_{0}\right)$ (red squares) and $\left(T(0), T_{z}(0)\right)=\left(20 T_{0}, 20 T_{0}\right)$ (blue diamonds) are plotted in a wider scaled, finding that $T_{z}^{(H)}(T) \approx T_{z, s}+q\left(T-T_{s}\right)$ (solid line) is reliable even till $T \sim 6 T_{0}$. The universal curve in the free cooling case is also plotted (dashed line) finding that, in effect, $q \sim q_{f}$. Similar results are found for other values of the parameters.

Finally, let us consider two systems, $A$ and $B$ with the same values of the parameters, but prepared in such a way that $\delta T_{B}(0)>\delta T_{A}(0)>0\left(\delta T_{B}(0)<\delta T_{A}(0)<0\right)$. Is it possible that, still, system $B$ reaches the stationary state faster than system $A$ ? In the linear regime, the question can be tackled with equation (40) by choosing the appropriate $\delta T_{z, A}(0)$ and $\delta T_{z, B}(0)$. A necessary condition for the effect is that the two curves cross each other at some time, $s>0$, which occurs if

$$
\mathrm{e}^{\left(\lambda_{1}-\lambda_{2}\right) s}=-\frac{u_{21}}{u_{11}} \frac{v_{21}+v_{22} \frac{\Delta T_{z}}{\Delta T}}{v_{11}+v_{12} \frac{\Delta T_{z}}{\Delta T}},
$$

where we have introduced $\Delta T \equiv \delta T_{B}(0)-\delta T_{A}(0)$ and $\Delta T_{z} \equiv \delta T_{z, B}(0)-\delta T_{z, A}(0)$. Note that equation (47) depends only on one single parameter $\frac{\Delta T_{z}}{\Delta T}$. For the studied values of the parameters, it is found that equation (47) has no solution for $\frac{\Delta T_{z}}{\Delta T}>0$ and has one 
Homogeneous dynamics in a vibrated granular monolayer

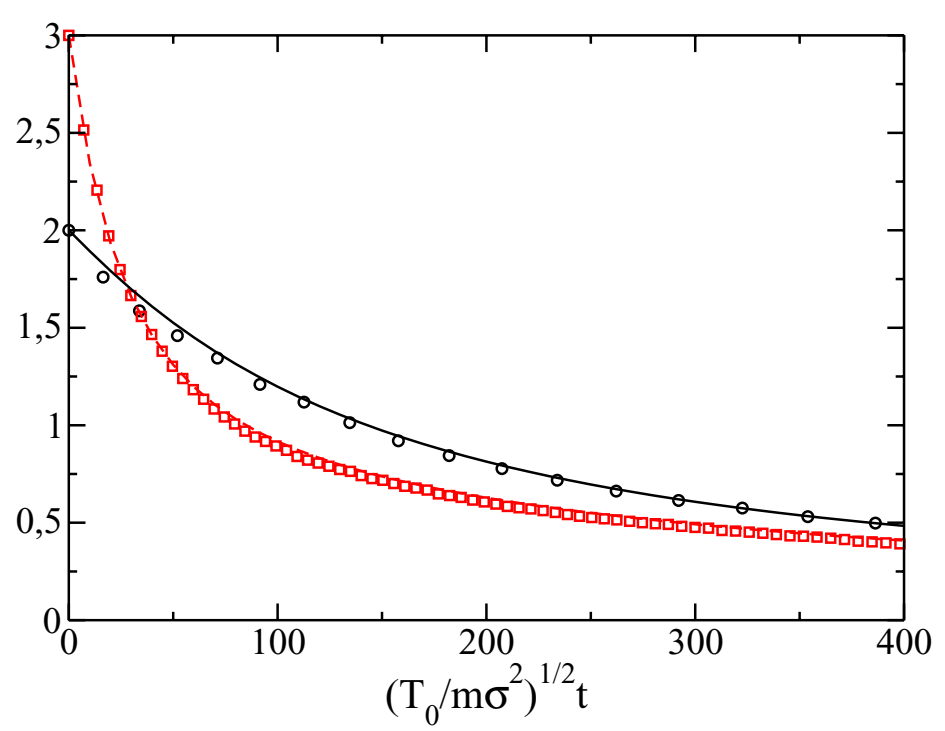

Figure 11. Time evolution of the horizontal temperature for $\epsilon=0.5, \alpha=0.9$ and two different initial conditions (color online), $\left(T_{A}(0), T_{z, A}(0)\right)=\left(2 T_{0}, 3.5 T_{0}\right)$ (black circles) and $\left(T_{B}(0), T_{z, B}(0)\right)=\left(3 T_{0}, 0.5 T_{0}\right)$ (red squares). The (black) solid line and (red) dashed line are their corresponding theoretical predictions.

solution for $\frac{\Delta T_{z}}{\Delta T}<0$. In any case, even if the curves cross each other, it can happen that the initially hotter system crosses the stationary value and reaches the stationary state less quickly. By analyzing the equations (31) and (32), it can be shown qualitatively that the condition $\frac{\Delta T_{z}}{\Delta T}<0$ can work also in the non-linear regime. This is because the abovementioned condition implies that the hottest initial configuration will cool down much quicker than the coolest one. In figure 11 the time evolution of the horizontal temperature is plotted for $\epsilon=0.5, \alpha=0.9$ and two different initial conditions (color online), $\left(T_{A}(0), T_{z, A}(0)\right)=\left(2 T_{0}, 3.5 T_{0}\right)$ (black circles) and $\left(T_{B}(0), T_{z, B}(0)\right)=\left(3 T_{0}, 0.5 T_{0}\right)$ (red squares). The (black) solid line and (red) dashed line are their corresponding theoretical predictions, i.e. the numerical solution of equations (31) and (32) with the corresponding initial condition. It is $\frac{\Delta T_{z}}{\Delta T}<0$ and, in fact, the two curves cross each other. Note that we are far from the linear regime as $T_{s} \approx 0.18 T_{0}$ and $T_{z, s} \approx 0.45 T_{0}$. A similar effect has been studied previously in the context of granular systems [41, 42]. In any case, it is important to remark that the effect does not contradict the hydrodynamic behavior as it occurs in the kinetic time scale.

\section{Discussion and conclusions}

In this paper we have proposed a closed dynamical equation for the one-particle distribution function for a system of inelastic hard spheres confined between two parallel hard flat plates valid in the low density limit. The distance between the walls is smaller than twice the diameter of the particles so that the system is actually Q2D, and the bottom plate is a sawtooth wall that injects energy into the system. The structure of the equation is similar to the 'traditional' Boltzmann equation: it contains a free 
streaming contribution, a collisional term and a wall term. The collisional contribution takes into account the effect of the confinement because, given a tagged particle, only collisions in some directions are possible. The wall contribution depends on the nature of the wall, concretely on the wall-particle collision rule. From the kinetic equation and assuming that the system is spatially homogeneous, we have derived the evolution equations for the horizontal and vertical temperatures. For the derivation, it has been assumed that the one-particle distribution function is a Gaussian with two temperatures (the horizontal and vertical ones) and that $\epsilon \equiv \frac{H-\sigma}{\sigma} \ll 1$. A very good agreement between the theoretical predictions for the temperatures and MD simulation results is found for a wide range of inelasticities if $\epsilon \leqslant 0.5$ without any fitting parameter. This good agreement is obtained not only for the stationary values, but also for the whole dynamics of the temperatures. It has been shown that, under certain conditions, the system with a larger initial granular temperature cools down more quickly and reaches the stationary state before than another system with an initial condition closer to the stationary state. Actually, it seems that the experimental realization of this effect is easy in a vibrated granular monolayer. The reason is that the relevant parameters are the horizontal and vertical temperatures that are easily controlled experimentally (the vertical temperature can be changed just by changing the parameters of the vibrating wall). Other memory effects such as the Kovacs effect [44] can also be studied in the context of our model. In contrast to other granular systems [45], the horizontal temperature always has an anomalous behavior because the horizontal temperature equation does not contain $v_{p}$ and a sudden change of it does not change the cooling/ heating rate.

Remarkably, it is found that, independently of the initial condition, after a transient, the system reaches a universal regime in which the two temperatures are related, i.e. the vertical temperature is a function of the horizontal temperature, $T_{z}^{(H)}(T)$, or vice versa, $T^{(H)}\left(T_{z}\right)$. This is the so-called homogeneous hydrodynamic regime. Numerical simulation results show that, in this regime, the relation between the temperatures is approximately linear and can be written in the form, $T_{z}^{(H)}(T) \approx T_{z, s}+p\left(T-T_{s}\right)$, even for temperatures for which the linearized equations are not expected to be reliable. Hence, in the homogeneous hydrodynamic regime, the following approximated closed equation for the horizontal temperature is obtained

$$
\frac{\mathrm{d} T}{\mathrm{~d} s}=\left[-(1-\alpha)+\frac{\epsilon^{2}}{12}[-(5 \alpha-1)+(3 \alpha+1) p]\right] T+\frac{\epsilon^{2}(3 \alpha+1)}{12} T_{s} .
$$

The study of the homogeneous hydrodynamic regime is relevant, as it is the first step to be done for the study of hydrodynamic in the plane. Here, by hydrodynamic in the plane we mean a closed description of the system in terms of the local $2 \mathrm{D}$ density, projected flow velocity on the plane and horizontal temperature. Actually, in the present model, the study of homogeneous hydrodynamic is specially relevant from a quantitative point of view as compared to other models. In effect, in the stochastic thermostat model or in the $\Delta$ model the one-particle distribution function in the homogeneous hydrodynamic regime is always close to a Gaussian, the deviation from it described by the kurtosis, $a_{2}$, which is always very small $[43,46]$. The transport coefficients, as calculated by the Chapman-Enskog scheme or by linear response methods, depend on the dynamics of the one-particle distribution function in the homogeneous hydrodynamic regime and, 
specifically, on the dynamics of $a_{2}[24,25,47]$. As $a_{2} \ll 1$, its effect on the transport coefficients is also very small and the Gaussian approximation (with one temperature) is a good approximation. In contrast, in our model there is not a small parameter in the one-particle distribution function, the one-temperature Gaussian approximation is not a good approximation and the effects of homogeneous hydrodynamics encoded in $T_{z}^{(H)}(T)$ are expected to be relevant for the computation of the transport coefficients.

Finally, let us mention that the kinetic equation can be extended to higher densities by using the Enskog approximation, i.e. assuming that there are not velocity correlations between colliding particles, although spatial correlations are taken into account through the pair correlation function at contact [37]. The situation in this case is more complex as it is not clear that the system can be considered to be homogeneous in the vertical direction and, in addition, it is possible that the dependence on the orientation of the pair correlation function can be relevant for the analysis. Work in these lines is in progress.

\section{Acknowledgments}

This research was supported by the Ministerio de Educación, Industria y Competitividad (Spain) through Grant No. FIS2017-87117-P (partially financed by FEDER funds).

\section{Appendix. Evaluation of some collisional integrals}

The objective of the appendix is to evaluate the collisional integrals that appears in the equations of the temperatures in the two-temperatures Gaussian approximation given by equation (25). The following property of the collision operator will be used

$$
\begin{aligned}
& \int_{\sigma / 2}^{H-\sigma / 2} \mathrm{~d} z \int \mathrm{d} \mathbf{v} \psi(\mathbf{v}) J_{z}[f \mid f] \\
& =\frac{\sigma^{2}}{2} \int \mathrm{d} \mathbf{v}_{1} \int \mathrm{d} \mathbf{v}_{2} f\left(\mathbf{v}_{1}\right) f\left(\mathbf{v}_{2}\right) \int_{\sigma / 2}^{H-\sigma / 2} \mathrm{~d} z \int_{\Omega(z)} \mathrm{d} \hat{\boldsymbol{\sigma}} \Theta\left(\mathbf{v}_{12} \cdot \hat{\boldsymbol{\sigma}}\right)\left|\mathbf{v}_{12} \cdot \hat{\boldsymbol{\sigma}}\right|\left(b_{\hat{\boldsymbol{\sigma}}}-1\right)\left[\psi\left(\mathbf{v}_{1}\right)+\psi\left(\mathbf{v}_{2}\right)\right] .
\end{aligned}
$$

Taking into account equation (A.1), the integral that appears in the vertical temperature equation is

$$
\begin{aligned}
& \frac{1}{H-\sigma} \int_{\sigma / 2}^{H-\sigma / 2} \mathrm{~d} z \int \mathrm{d} \mathbf{v}\left(v_{x}^{2}+v_{y}^{2}\right) J_{z}[f \mid f] \\
& =\frac{\sigma^{2}}{2(H-\sigma)} \int \mathrm{d} \mathbf{v}_{1} \int \mathrm{d} \mathbf{v}_{2} f\left(\mathbf{v}_{1}\right) f\left(\mathbf{v}_{2}\right) \int_{\sigma / 2}^{H-\sigma / 2} \mathrm{~d} z \int_{\Omega(z)} \mathrm{d} \hat{\boldsymbol{\sigma}} \Theta\left(\mathbf{v}_{12} \cdot \hat{\boldsymbol{\sigma}}\right)\left|\mathbf{v}_{12} \cdot \hat{\boldsymbol{\sigma}}\right|\left(b_{\hat{\boldsymbol{\sigma}}}-1\right)\left(v_{1 x}^{2}+v_{1 y}^{2}+v_{2 x}^{2}+v_{2 y}^{2}\right) .
\end{aligned}
$$

And using the collision rule, equations (1) and (2), we have

$\left(b_{\hat{\boldsymbol{\sigma}}}-1\right)\left(v_{1 x}^{2}+v_{1 y}^{2}+v_{2 x}^{2}+v_{2 y}^{2}\right)=-(1+\alpha)(\hat{\boldsymbol{\sigma}} \cdot \mathbf{g})\left(g_{x} \hat{\sigma}_{x}+g_{y} \hat{\sigma}_{y}\right)+\frac{(1+\alpha)^{2}}{2}(\hat{\boldsymbol{\sigma}} \cdot \mathbf{g})^{2}\left(\hat{\sigma}_{x}^{2}+\hat{\sigma}_{y}^{2}\right)$,

where we changed the notation of the relative velocity, $\mathbf{g} \equiv \mathbf{v}_{12}$, for simplicity. Then, equation (A.2) can be written as 


$$
\begin{aligned}
& \frac{1}{H-\sigma} \int_{\sigma / 2}^{H-\sigma / 2} \mathrm{~d} z \int \mathrm{d} \mathbf{v}\left(v_{x}^{2}+v_{y}^{2}\right) J_{z}[f \mid f]=\frac{\sigma^{2}}{2(H-\sigma)} \int \mathrm{d} \mathbf{v}_{1} \int \mathrm{d} \mathbf{v}_{2} f\left(\mathbf{v}_{1}\right) f\left(\mathbf{v}_{2}\right) \int_{\sigma / 2}^{H-\sigma / 2} \mathrm{~d} z \\
& \int_{\Omega(z)} \mathrm{d} \hat{\boldsymbol{\sigma}} \Theta(\mathbf{g} \cdot \hat{\boldsymbol{\sigma}})|\mathbf{g} \cdot \hat{\boldsymbol{\sigma}}|\left[-(1+\alpha)(\hat{\boldsymbol{\sigma}} \cdot \mathbf{g})\left(g_{x} \hat{\sigma}_{x}+g_{y} \hat{\sigma}_{y}\right)+\frac{(1+\alpha)^{2}}{2}(\hat{\boldsymbol{\sigma}} \cdot \mathbf{g})^{2}\left(\hat{\sigma}_{x}^{2}+\hat{\sigma}_{y}^{2}\right)\right] \\
& =\frac{\sigma^{2}}{2(H-\sigma)} \int \mathrm{d} \mathbf{v}_{1} \int \mathrm{d} \mathbf{v}_{2} f\left(\mathbf{v}_{1}\right) f\left(\mathbf{v}_{2}\right) \int_{\sigma / 2}^{H-\sigma / 2} \mathrm{~d} z \\
& \int_{\Omega(z)} \mathrm{d} \hat{\boldsymbol{\sigma}} \Theta(-\mathbf{g} \cdot \hat{\boldsymbol{\sigma}})|\mathbf{g} \cdot \hat{\boldsymbol{\sigma}}|\left[-(1+\alpha)(\hat{\boldsymbol{\sigma}} \cdot \mathbf{g})\left(g_{x} \hat{\sigma}_{x}+g_{y} \hat{\sigma}_{y}\right)+\frac{(1+\alpha)^{2}}{2}(\hat{\boldsymbol{\sigma}} \cdot \mathbf{g})^{2}\left(\hat{\sigma}_{x}^{2}+\hat{\sigma}_{y}^{2}\right)\right],
\end{aligned}
$$

where we have changed $\mathbf{v}_{1}$ by $\mathbf{v}_{2}$ in the last step. Hence, we can get rid of the $\Theta$ function and we have

$\frac{1}{H-\sigma} \int_{\sigma / 2}^{H-\sigma / 2} \mathrm{~d} z \int \mathrm{d} \mathbf{v}\left(v_{x}^{2}+v_{y}^{2}\right) J_{z}[f \mid f]=\frac{1}{H-\sigma} \int_{\sigma / 2}^{H-\sigma / 2} \mathrm{~d} z\left[\frac{(1+\alpha)^{2}}{2} H_{1}(z)-(1+\alpha) H_{2}(z)\right]$,

where

$$
\begin{aligned}
& H_{1}(z)=\frac{\sigma^{2}}{4} \int \mathrm{d} \mathbf{v}_{1} \int \mathrm{d} \mathbf{v}_{2} f\left(\mathbf{v}_{1}\right) f\left(\mathbf{v}_{2}\right) \int_{\Omega(z)} \mathrm{d} \hat{\boldsymbol{\sigma}}|\mathbf{g} \cdot \hat{\boldsymbol{\sigma}}|^{3}\left(\hat{\sigma}_{x}^{2}+\hat{\sigma}_{y}^{2}\right), \\
& H_{2}(z)=\frac{\sigma^{2}}{4} \int \mathrm{d} \mathbf{v}_{1} \int \mathrm{d} \mathbf{v}_{2} f\left(\mathbf{v}_{1}\right) f\left(\mathbf{v}_{2}\right) \int_{\Omega(z)} \mathrm{d} \hat{\boldsymbol{\sigma}}|\mathbf{g} \cdot \hat{\boldsymbol{\sigma}}|(\mathbf{g} \cdot \hat{\boldsymbol{\sigma}})\left(g_{x} \hat{\sigma}_{x}+g_{y} \hat{\sigma}_{y}\right) .
\end{aligned}
$$

Let us first calculate $H_{1}$. To do it, let us perform the following change of variables

$$
\begin{aligned}
& \mathbf{C}=\frac{1}{2}\left(\mathbf{c}_{1}+\mathbf{c}_{2}\right), \\
& \mathbf{c}=\mathbf{c}_{1}-\mathbf{c}_{2},
\end{aligned}
$$

where

$$
\mathbf{c}_{i}=\frac{1}{w}\left(v_{i, x} \mathbf{e}_{x}+v_{i, y} \mathbf{e}_{y}\right)+\frac{v_{i, z}}{w_{z}} \mathbf{e}_{z},
$$

for $i=1,2$. Taking into account the expression of the distribution function given by equation (25)

$$
f\left(\mathbf{v}_{1}\right) f\left(\mathbf{v}_{2}\right)=\frac{n^{2}}{\pi^{3} w^{4} w_{z}^{2}} \mathrm{e}^{-2 C^{2}} \mathrm{e}^{-\frac{c^{2}}{2}},
$$

we have

$H_{1}(z)=\frac{n^{2} \sigma^{2}}{4 \pi^{3}} \int \mathrm{d} \mathbf{C} \mathrm{e}^{-2 C^{2}} \int \mathrm{d} \mathbf{c} \mathrm{e}^{-\frac{c^{2}}{2}} \int_{\Omega(z)} \mathrm{d} \hat{\boldsymbol{\sigma}}|\mathbf{c} \cdot \mathbf{a}|^{3}\left(w^{2} \hat{\sigma}_{x}^{2}+w^{2} \hat{\sigma}_{y}^{2}+w_{z}^{2} \hat{\sigma}_{z}^{2}\right)^{3 / 2}\left(\hat{\sigma}_{x}^{2}+\hat{\sigma}_{y}^{2}\right)$,

where we have introduced the unit vector

$$
\mathbf{a} \equiv \frac{w \hat{\sigma}_{x} \mathbf{e}_{x}+w \hat{\sigma}_{y} \mathbf{e}_{y}+w_{z} \hat{\sigma}_{z} \mathbf{e}_{z}}{\sqrt{w^{2} \hat{\sigma}_{x}^{2}+w^{2} \hat{\sigma}_{y}^{2}+w_{z}^{2} \hat{\sigma}_{z}^{2}}} .
$$


The velocity integrals in $\mathbf{C}$ and $\mathbf{c}$ are

$$
\int \mathrm{d} \mathbf{C e}^{-2 C^{2}}=\frac{\pi^{3 / 2}}{2 \sqrt{2}}, \quad \int \mathrm{d} \mathbf{c} \mathrm{e}^{-\frac{c^{2}}{2}}\left|c_{x}\right|^{3}=8 \pi,
$$

so that, by symmetry, it is

$$
H_{1}(z)=\frac{n^{2} \sigma^{2}}{\sqrt{2 \pi}} \int_{\Omega(z)} \mathrm{d} \hat{\boldsymbol{\sigma}}\left(w^{2} \hat{\sigma}_{x}^{2}+w^{2} \hat{\sigma}_{y}^{2}+w_{z}^{2} \hat{\sigma}_{z}^{2}\right)^{3 / 2}\left(\hat{\sigma}_{x}^{2}+\hat{\sigma}_{y}^{2}\right),
$$

or, by introducing the parametrization of $\Omega(z)$ given by equation (9)

$$
H_{1}(z)=\sqrt{2 \pi} n^{2} \sigma^{2} \int_{\frac{\pi}{2}-b_{2}(z)}^{\frac{\pi}{2}+b_{1}(z)} \mathrm{d} \theta \sin ^{3} \theta\left(w^{2} \sin ^{2} \theta+w_{z}^{2} \cos ^{2} \theta\right)^{3 / 2} .
$$

Changing variables to $z_{1}$ defined by equation (16) and integrating also in $z$, we obtain

$$
\begin{array}{r}
\int_{\sigma / 2}^{H-\sigma / 2} \mathrm{~d} z H_{1}(z)=\sqrt{2 \pi} n^{2} \sigma \int_{\sigma / 2}^{H-\sigma / 2} \mathrm{~d} z \int_{\sigma / 2}^{H-\sigma / 2} \mathrm{~d} z_{1}\left[1-\left(\frac{z_{1}-z}{\sigma}\right)^{2}\right] \\
\left\{w^{2}\left[1-\left(\frac{z_{1}-z}{\sigma}\right)^{2}\right]+w_{z}^{2}\left(\frac{z_{1}-z}{\sigma}\right)^{2}\right\}^{3 / 2},
\end{array}
$$

or, in terms of the dimensionless variables

$$
\begin{gathered}
\tilde{z}_{1}=\frac{z_{1}-\sigma / 2}{\sigma}, \quad \tilde{z}_{2}=\frac{z-\sigma / 2}{\sigma} \\
\int_{\sigma / 2}^{H-\sigma / 2} \mathrm{~d} z H_{1}(z)=2 \sqrt{2 \pi} n^{2} \sigma^{3} \int_{0}^{\epsilon} \mathrm{d} \tilde{z}_{1} \int_{0}^{\tilde{z}_{1}} \mathrm{~d} \tilde{z}_{2}\left(1-\tilde{z}_{12}\right)^{2}\left[w^{2}\left(1-\tilde{z}_{12}^{2}\right)+w_{z}^{2} \tilde{z}_{12}^{2}\right]^{3 / 2}
\end{gathered}
$$

where we have used the integrand as invariant under the change $\tilde{z}_{1}$ by $\tilde{z}_{2}$ and we have introduced $\tilde{z}_{12} \equiv \tilde{z}_{1}-\tilde{z}_{2}$. Finally, changing variables to

$$
y=\tilde{z_{1}}-\tilde{z}_{2}, \quad Y=\frac{1}{2}\left(\tilde{z_{1}}+\tilde{z}_{2}\right),
$$

it is obtained

$$
\begin{aligned}
\int_{\sigma / 2}^{H-\sigma / 2} \mathrm{~d} z H_{1}(z)= & 2 \sqrt{2 \pi} n^{2} \sigma^{3} \int_{0}^{\epsilon} \mathrm{d} y \int_{y / 2}^{\epsilon-y / 2} \mathrm{~d} Y\left(1-y^{2}\right)\left[w^{2}\left(1-y^{2}\right)+w_{z}^{2} y^{2}\right]^{3 / 2} \\
= & 2 \sqrt{2 \pi} n^{2} \sigma^{3} \int_{0}^{\epsilon} \mathrm{d} y(\epsilon-y)\left(1-y^{2}\right)\left[w^{2}\left(1-y^{2}\right)+w_{z}^{2} y^{2}\right]^{3 / 2} .
\end{aligned}
$$

The evaluation of $\mathrm{H}_{2}(z)$ follows similar lines. Changing variables to $\mathbf{C}$ and $\mathbf{c}$ introduced in equations (A.8) and (A.9) and integrating in C, we have

$H_{2}(z)=\frac{n^{2} \sigma^{2} w}{8 \sqrt{2} \pi^{3 / 2}} \int \mathrm{d} \mathbf{c} \mathrm{e}^{-\frac{c^{2}}{2}} \int_{\Omega(z)} \mathrm{d} \hat{\boldsymbol{\sigma}}|\mathbf{c} \cdot \mathbf{a}|(\mathbf{c} \cdot \mathbf{a})\left(w^{2} \hat{\sigma}_{x}^{2}+w^{2} \hat{\sigma}_{y}^{2}+w_{z}^{2} \hat{\sigma}_{z}^{2}\right)\left(c_{x} \hat{\sigma}_{x}+c_{y} \hat{\sigma}_{y}\right)$.

Expressing $c_{x} \hat{\sigma}_{x}+c_{y} \hat{\sigma}_{y}$ in terms of the components of $\mathbf{c}$ in the orthonormal basis $\left\{\mathbf{a}, \mathbf{b}_{1}, \mathbf{b}_{2}\right\}$ and integrating in $\mathbf{c}$, we have 


$$
H_{2}(z)=\frac{n^{2} \sigma^{2} w}{\sqrt{2 \pi}} \int_{\Omega(z)} \mathrm{d} \hat{\boldsymbol{\sigma}}\left(w^{2} \hat{\sigma}_{x}^{2}+w^{2} \hat{\sigma}_{y}^{2}+w_{z}^{2} \hat{\sigma}_{z}^{2}\right)\left(\hat{\sigma}_{x} \mathbf{e}_{x} \cdot \mathbf{a}+\hat{\sigma}_{y} \mathbf{e}_{y} \cdot \mathbf{a}\right),
$$

where we have used that, by symmetry, $\int \mathrm{d} \mathbf{c} \mathrm{e}^{-\frac{c^{2}}{2}}|\mathbf{c} \cdot \mathbf{a}|(\mathbf{c} \cdot \mathbf{a})\left(\mathbf{c} \cdot \mathbf{b}_{i}\right)=0$, for $i=1,2$. Now, writing the explicit expression of $\mathbf{e}_{i} \cdot \mathbf{a}$ with the aid of equation (A.13), we have

$$
H_{2}(z)=\frac{n^{2} \sigma^{2} w^{2}}{\sqrt{2 \pi}} \int_{\Omega(z)} \mathrm{d} \hat{\boldsymbol{\sigma}}\left(w^{2} \hat{\sigma}_{x}^{2}+w^{2} \hat{\sigma}_{y}^{2}+w_{z}^{2} \hat{\sigma}_{z}^{2}\right)\left(\hat{\sigma}_{x}^{2}+\hat{\sigma}_{y}^{2}\right) .
$$

Finally, following exactly the same steps as above, it is obtained

$$
\int_{\sigma / 2}^{H-\sigma / 2} \mathrm{~d} z H_{2}(z)=2 \sqrt{2 \pi} n^{2} \sigma^{3} w^{2} \int_{0}^{\epsilon} \mathrm{d} y(\epsilon-y)\left(1-y^{2}\right) \sqrt{w^{2}\left(1-y^{2}\right)+w_{z}^{2} y^{2}} .
$$

Using equations (A.5), (A.21) and (A.25), the expression for $\frac{1}{H-\sigma} \int_{\sigma / 2}^{H-\sigma / 2} \mathrm{~d} z \int \mathrm{d} \mathbf{v} \frac{m}{2}\left(v_{x}^{2}+v_{y}^{2}\right) J_{z}[f \mid f]$ given by equation (28) is obtained. The evaluation of $\frac{1}{H-\sigma} \int_{\sigma / 2}^{H-\sigma / 2} \mathrm{~d} z \int \mathrm{d} \mathbf{v} \frac{m}{2} v_{z}^{2} J_{z}[f \mid f]$ follows exactly the same steps.

Finally, the particle-wall collisional integral is

$$
\begin{array}{r}
\frac{m}{2(H-\sigma)} \int \mathrm{d} \mathbf{v} v_{z}^{2} L_{s} f(\mathbf{v})=\frac{m}{2(H-\sigma)} \int \mathrm{d} \mathbf{v} \theta\left(-v_{z}\right)\left|v_{z}\right|\left(4 v_{p}^{2}-4 v_{p} v_{z}\right) f(\mathbf{v}) \\
=\frac{m}{2(H-\sigma)} n v_{p} w_{z}\left(\frac{2}{\sqrt{\pi}} v_{p}+w_{z}\right)
\end{array}
$$

where the explicit form of the velocity distribution given by equation (25) has been used. Taking into account that, in order to be valid for the homogeneous approximation, the condition $v_{p} \ll w_{z}$ has to be fulfilled, thereby we obtain the expression of the main text.

\section{References}

[1] Campbell C S 1990 Rapid granular flows Annu. Rev. Fluid Mech. 2257

[2] Goldhirsch I 2003 Rapid granular flows Annu. Rev. Fluid Mech. 35267

[3] Aranson I S and Tsimring L S 2006 Patterns and collective behavior in granular media: theoretical concepts Rev. Mod. Phys. 78641

[4] Brey J J, Dufty J W, Kim C S and Santos A 1998 Hydrodynamics for a granular flow at low density Phys. Rev. E 584638

[5] Garzó V and Dufty J W 1999 Dense fluid transport for inelastic hard spheres Phys. Rev. E 595895

[6] Livne E, Meerson B and Sasorov P V 2002 Symmetry-breaking instability and strongly peaked periodic clustering states in a driven granular gas Phys. Rev. E 65021302

[7] Brey J J, Ruiz-Montero M J, Moreno F and García-Rojo R 2002 Transversal inhomogeneities in dilute vibrofluidized granular fluids Phys. Rev. E 65061302

[8] Khain E and Meerson B 2004 Oscillatory instability in a driven granular gas Europhys. Lett. 65193

[9] Khain E and Meerson B 2003 Onset of thermal convection in a horizontal layer of granular gas Phys. Rev. E 67021306

[10] Melby P, Vega Reyes F, Prevost A, Robertson R, Kumar P, Egolf D A and Urbach J S 2005 The dynamics of thin vibrated granular layers J. Phys.: Condens. Matter 17 S2689-704

[11] Mujica N and Soto R 2016 Dynamics of noncoheisve confined granular media Recent Advances in Fluid Dynamics with Enviromental Applications ed J Klapp et al (New York: Springer) pp 445-63 
[12] Olafsen J S and Urbach J S 1998 Clustering, order, and collapse in a driven granular monolayer Phys. Rev. Lett. $\mathbf{8 1} 4369$

[13] Losert W, Cooper D G W and Gollub J P 1999 Propagating front in an excited granular layer Phys. Rev. E 595855

[14] Prevost A, Egolf D A and Urbach J S 2002 Forcing and velocity correlations in a vibrated granular monolayer Phys. Rev. Lett. 89084301

[15] Olafsen J S and Urbach J S 2005 Two-dimensional melting far from equilibrium in a granular monolayer Phys. Rev. Lett. 95098002

[16] Roeller K, Clewett J P D, Bowley R M, Herminghaus S and Swift M R 2011 Liquid-gas phase separation in confined vibrated dry granular matter Phys. Rev. Lett. 107048002

[17] Clewett J P D, Roeller K, Bowley R M, Herminghaus S and Swift M R 2012 Emergent surface tension in vibrated, noncohesive granular media Phys. Rev. Lett. 109228002

[18] Castillo G, Mújica N and Soto R 2012 Fluctuations and criticality of a granular solid-liquid-like phase transition Phys. Rev. Lett. 109095701

[19] Néel B, Rondini I, Turzillo A, Mujica N and Soto R 2014 Dynamics of a first-order transition to an absorbing state Phys. Rev. E 89042206

[20] Castillo G, Mújica N and Soto R 2015 Universality and criticality of a second-order granular solid-liquid-like phase transition Phys. Rev. E 91012141

[21] Williams D R M and MacKintosh F C 1996 Driven granular media in one dimension: correlations and equation of state Phys. Rev. E 54 R9

[22] Brito R, Risso D and Soto R 2013 Hydrodynamic modes in a confined granular fluid Phys. Rev. E 87022209

[23] Brey J J, García de Soria M I, Maynar P and Buzón V 2014 Memory effects in the relaxation of a confined granular gas Phys. Rev. E 90032207

[24] García de Soria M I, Maynar P and Trizac E 2013 Linear hydrodynamics for driven granular gases Phys. Rev. E 87022201

[25] Garzó V, Chamorro M G and Vega Reyes F 2013 Transport properties for driven granular fluids in situations close to homogeneous steady states Phys. Rev. E 87032201

[26] Brey J J, Buzón V, García de Soria M I and Maynar P 2016 Stability analysis of the homogeneous hydrodynamics of a model for a confined granular gas Phys. Rev. E 93062907

[27] Cafiero R, Luding S and Herrmann H J 2000 Two-dimensional granular gas of inelastic spheres with multiplicative driving Phys. Rev. Lett. 846014

[28] Risso D, Soto R and Guzmán M 2018 Effective two-dimensional model for granular matter with phase separation Phys. Rev. E 98022901

[29] Brey J J, Maynar P and García de Soria M I 2016 Kinetic equation and nonequilibrium entropy for a quasitwo-dimensional gas Phys. Rev. E 94040103

[30] Brey J J, García de Soria M I and Maynar P 2017 Boltzmann kinetic equation for a strongly confined gas of hard spheres Phys. Rev. E 96042117

[31] Schmidt M and Löwen H 1997 Phys. Rev. E 557228

[32] Maynar P, García de Soria M I and Brey J J 2019 Understanding an instability in vibrated granular monolayers Phys. Rev. E 99032903

[33] Brey J J, García de Soria M I and Maynar P 2019 (in preparation)

[34] Résibois P and De Leener M 1977 Classical Kinetic Theory of Fluids (New York: Wiley)

[35] McLennan J A 1989 Introduction to Non-equilibrium Statistical Mechanics (Englewood Cliffs, NJ: Prentice Hall)

[36] Dorfman J R and van Beijeren H 1977 Statistical Mechanics, Part B ed B J Berne (New York: Plenum)

[37] Maynar P, García de Soria M I and Brey J J 2018 The Enskog equation for confined elastic hard spheres J. Stat. Phys. 170 999-1018

[38] Brey J J and Ruiz-Montero M J 2009 Vibrated granular gas confined by a piston Phys. Rev. E 79031305

[39] van Noije T P C and Ernst M H 1998 Velocity distribution in homogeneous granular fluids: the free and the heated case Granul. Matter 157

[40] Allen M P and Tisdesley D J 1987 Computer Simulations of Liquids (New York: Oxford Science Publications)

[41] Lasanta A, Vega Reyes F, Prados A and Santos A 2017 Whe the hotter cools more quickly: Mpemba effect in granular fluids Phys. Rev. Lett. 119148001

[42] Torrente A, López-Castaño M A, Lasanta A, Vega Reyes F, Prados A and Santos A 2019 Large Mpemba-like effect in a gas of inelastic rough hard spheres (arXiv:1902.09404v1) 
[43] García de Soria M I, Maynar P and Trizac E 2012 Universal reference state in a driven homogeneous granular gas Phys. Rev. E 85051301

[44] Kovacs A J 1963 Adv. Polym. Sci. 3394

[45] Prados A and Trizac E 2014 Kovacs-like memory effect in driven granular gases Phys. Rev. Lett. 112198001

[46] Brey J J, Maynar P, García de Soria M I and Buzón V 2014 Homogeneous hydrodynamics of a collisional model of confined granular gases Phys. Rev. E 89052209

[47] Brey J J, Buzón V, Maynar P and García de Soria M I 2015 Hydrodynamics for a model of a confined quasitwo-dimensional granular gas Phys. Rev. E 91052201 